\title{
Problemi ellittici in un angolo $(*)$.
}

\author{
Gumo Trombetiti (Napoli)
}

\begin{abstract}
Summary. - This paper is concerned with boundary value problems for linear partial differential equations of elliptic type in a plane sector; in the first part of the paper Sobolev weight spaces have been studied and embedding and trace theorems have been proved; in the second part it is proved that problem is index type.
\end{abstract}

Detto $A$ l'angolo aperto di $R^{2}$ con vertice nell'origine e delimitato dalle semirette $\sigma_{1}$ e $\sigma_{2}$, consideriamo il problema:

$$
\begin{array}{ll}
A u=f & \text { in } A, \\
B_{j k} u=g_{j k} & \text { su } \sigma_{k}, j=1, \ldots, m ; k=1,2,
\end{array}
$$

dove $A$ è un operatore difierenziale lineare di ordine $2 m$ propriamente ellittico in $\bar{A}$ ed all'infinito, i $B_{j k}$ sono operatori differenziali di frontiera su $\sigma_{k}$ verificanti la condizione complementare rispetto ad $A$ su $\bar{\sigma}_{k}$ ed all'infinito.

A. Avantagaiati e M. Troisi in [1], [2], [3] hanno studiato tale problema in spazi di Sobolev con doppio peso $W_{s_{1}, s_{2}}^{r}(\Lambda)$, dove i pesi hanno il ruolo di prescrivere il comportamento nell'origine ed all'infinito delle funzioni di $W_{s_{2}, s_{2}}^{r}(\Lambda)$.

Tali autori hanno dimostrato che, sotto opportune ipotesi ed esclusi certi valori eccezionali di $s_{i}-r+1$, il problema in questi spazi è ad indice.

Va rilevato che gli spazi $W_{s_{1}, s_{2}}^{r}(\Lambda)$ hanno la proprietà che le eventuali tracce nel'origine di funzioni ad essi appartenenti e delle loro derivate si annullano.

Ciò comporta che tra le condizioni di compatibilità per il problema vi sono quelle che prescrivono ai termini noti ed alle loro derivate fino a quelle di un certo ordine di essere dotati di tracce nulle nell'origine.

Per svincolarsi da tali limitazioni i suddetti autori hanno introdotto un'altra classe di spazi denotati con $W_{s_{1}, s_{2}}^{* r}(\Lambda)$ a cui appartengono anche funzioni con le eventuali tracce nell'origine non nulle.

Hanno dimostrato che se $f$ e $g_{j k}$ sono prese in opportuni spazi funzionali ed inoltre verificano un numero finito di condizioni di compatibilità nell'origine il problema in $W_{s_{1}, s_{2}}^{* r}(\Lambda)$ si può ricondurre ad un altro in $W_{s_{1}, s_{2}}^{\tau}(\Lambda)$.

Per la risolubilità di quest'ultimo problema sarà poi necessario che siano soddisfatte altre condizioni di compatibilità in numero finito. Pertanto il numero totale

(*) Entrata in Redazione il 14 agosto 1974. 
delle condizioni di compatibilità necessarie e sufficienti per la risolubilità del problema in $W_{s_{1}, s_{2}}^{* r}(\Lambda)$ è finito ed il procedimento porta a stabilire che la eventuale soluzione dipende da un numero finito di costanti arbitrarie.

In questo lavoro mi propongo di riprendere lo studio del problema in un'altra classe di spazi di Soboler denotati con $U_{s_{1}, s_{2}}^{r}(\Lambda)\left(r \in N, s_{1} \geqslant 0\right)$ del tipo degli spazi $W_{s_{1}, s_{2}}^{* r}(A)$ nel caso in cui $s_{1}>0$, per giangere ad ottenere dei risultati che siano svincolati dal problema in $W_{s_{1}, s_{\mathrm{g}}}^{r}(\Lambda)$ studiato in [3], e in cui sia preso anche in considerazione il caso $s_{1}=0$ tralasciato in [3].

Ciò richiede un preliminare studio degli spazi $U_{s_{1}, s_{2}}^{r}(\Lambda)$ che implichi anche un ulteriore approfondimento degli spazi $W_{s_{1}, s_{2}}^{* r}(\Lambda)$ maggiore di quello ottenuto in [1] (in cui ad es. manca una caratterizzazione dei corrispondenti spazi di tracce).

Stabiliseo per tali spazi dei teoremi di immersione, di compattezza, di densità e di tracce sui lati e nel vertice di $A$.

Usufruendo di tale caratterizzazione degli spazi $U_{s_{1}, s_{\mathrm{a}}}^{r}(\Lambda)$ e dei corrispondenti spazi di tracce e poggiando sui risultati di [3] relativi al problema posto in $W_{s_{1}, s_{z}}^{r}(A)$, stabiliseo delle limitazioni a priori per il problema sopra citato e (nel caso $s_{1}>0$ ) per il suo aggiunto funzionale.

Da tali limitazioni si deduce che l'operatore associato al problema nel caso $s_{1}>0$ è ad indice, e nel caso $s_{1}=0$ ha nucleo di dimensione finita e codominio chiuso.

È da rilevare che questi spazi sono tali che se $u \in U_{s_{1}, s_{2}}^{r}(\Lambda)$ per ogni $a \in R_{+}$si ha:

$$
\sum_{|\alpha| \leqslant r-2} \int_{A \cap\{|\alpha| \leqslant a\}}\left|\partial^{\alpha} u\right|^{2} d x+\sum_{|\alpha|=r-1} \int_{A \cap\{|\alpha| \leqslant a\}}|x|^{\mid 2\left(s_{1}-1\right)}\left|\partial^{\alpha} u\right|^{2} d x+\sum_{|\alpha|=r} \int_{A \cap\{|x| \leqslant a\}}|x|^{2 s_{2}}\left|\partial^{\alpha} u\right|^{2} d x \leqslant+\infty .
$$

Il comportamento all'infinito è come in $W_{s_{1}, s_{2}}^{r}(\Lambda)$.

La formula di maggiorazione per le soluzioni del problema a cui pervengo è del tipo:

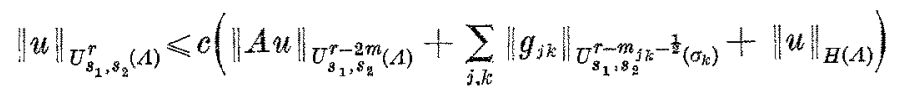

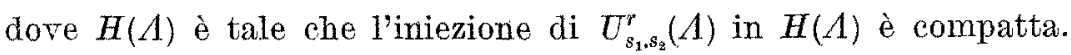

Essa è valida, esclusi valori eccezionali di $s_{i}-r+1$, se $s_{1} \in[0,1[$.

Analoga limitazione si dimostra se $s_{1} \in(] 0, \frac{3}{2}[-\{1\})$ per le eventuali soluzioni del problema aggiunto funzionale.

In una prima parte del lavoro, n. 1-7, approfondisco lo studio degli spazi $U_{s_{1}, s_{2}}^{r}(\Lambda)$.

Nel n. 8 enuncio le ipotesi per il problema ed i risultati che dimostro nel n. 9 .

Nel n. 10 stabilisco i risultati per il problema aggiunto.

\section{1. - Notaxioni e lemmi preliminari.}

Sia $A$ l'angolo aperto di $R^{3}$ con vertice nell'origine e delimitato dalle semirette $\sigma_{1}$ e $\sigma_{2}$ di anomalia $\theta_{1}$ e $\theta_{2}$ con:

$$
-\pi / 2<\theta_{1}<\theta_{2}<\frac{3}{2} \pi
$$


Detto $x=\left(x_{1}, x_{2}\right)$ il generico elemento di $R^{2}$ porremo:

$$
\Lambda_{a}=\{x \in A:|x|<a\}
$$

$\operatorname{con} a \in R_{+}$e $|x|=\sqrt{x_{1}^{2}+x_{2}^{2}}$.

Se $u(x)$ è una funzione definita in $A$ porremo:

$$
u_{\pi}(\varrho, \theta)=u(\varrho \cos \theta, \varrho \operatorname{sen} \theta)
$$

e se $\alpha=\left(\alpha_{1}, \alpha_{2}\right)$ è una coppia di interi positivi o nulli porremo:

$$
\partial^{\alpha}=\frac{\partial|\alpha|}{\partial x_{1}^{\alpha_{1}} \partial x_{2}^{\alpha_{2}}} \quad \text { ove }|\alpha|=\alpha_{1}+\alpha_{2} .
$$

Porremo ancora $\forall k \in N$ :

$$
\left|\partial^{k} u\right|^{2}=\sum_{|\alpha|=k}\left|\partial^{\alpha} u\right|^{2}
$$

Con il simbolo $x^{\alpha}$ ove $x \in R^{2}$ ed $\alpha \in N^{2}$ si intenderà :

$$
x^{\alpha}=x_{1}^{\alpha_{1}} x_{2}^{\alpha_{2}}
$$

Detta $g(\varrho)$ una funzione della sola variabile $\varrho$ si porrà:

$$
g^{(i)}(\varrho)=\frac{d^{i} g}{d \varrho^{i}}
$$

Diremo che $f(x) \in L^{\infty}(\Omega)$ se e solo se $f(x)$ è misurabile ed esiste $K \in R_{+}$tale che riesca

$$
|f(x)| \leqslant K \quad \text { quasi ovunque in } \Omega
$$

diremo che $f(x) \in L_{\alpha}^{\infty}(\Omega)$ se e solo se $|x|^{\alpha} f(x) \in L^{\infty}(\Omega)$.

Lemma 1.1. - Se $a \in R_{+}, \beta>-\frac{1}{2}$ e $\gamma \in R$ riesce:

$$
\begin{aligned}
\int_{0}^{a} \varrho^{2 \beta}|g(\varrho)|^{2} d \varrho \leqslant c\left(\int_{0}^{2 a} \varrho^{2(\beta+1)}\left|g^{\prime}(\varrho)\right|^{2} d \varrho\right. & \left.+\int_{a}^{2 a}|g(\varrho)|^{2} d \varrho\right) \leqslant \\
& \leqslant c_{\gamma}\left(\int_{0}^{2 a} \varrho^{2(\beta+1)}\left|g^{\prime}(\varrho)\right|^{2} d \varrho+\int_{0}^{2 a} \varrho^{2 \gamma}|g(\varrho)|^{2} d \varrho\right)
\end{aligned}
$$

per ogni funzione g per oui abbiano senso tali integrali.

7 - Annali di Hatematice 
Sia $\zeta \in \mathcal{D}([0,+\infty[)$ tale che:

$$
\zeta \begin{cases}=1 & \varrho \leqslant a \\ =0 & \varrho \geqslant 2 a .\end{cases}
$$

Posto:

$$
f(\varrho)=g(\varrho) \cdot \zeta(\varrho)
$$

riesce:

$$
\left.\int_{0}^{a}\left|\varrho^{2 \beta}\right| g(\varrho)\right|^{2} d \varrho \leqslant \int_{0}^{\infty} \varrho^{2 \beta}|f(\varrho)|^{2} d \varrho=\int_{0}^{\infty} \varrho^{2 \beta}\left|\int_{\varrho}^{\infty} f^{\prime}(t) d t\right|^{2} d \varrho .
$$

Applicando il Lemma 2.1, pag. 286 di [6] si ottiene:

$$
\int_{0}^{a} \varrho^{2 \beta}|g(\varrho)|^{2} d \varrho \leqslant c \int_{0}^{\infty} \varrho^{2(\beta+1)}\left|f^{\prime}(\varrho)\right|^{2} d \varrho \leqslant c\left(\int_{0}^{2 a} \varrho^{2(\beta+1)}\left|g^{\prime}(\varrho)\right|^{2} d \varrho+\int_{a}^{2 a}|g(\varrho)|^{2} d \varrho\right) .
$$

Osservando poi che:

$$
\int_{a}^{2 a}|g(\varrho)|^{2} d \varrho \leqslant c_{\gamma} \int_{a}^{2 a} \varrho^{2 \gamma}|g(\varrho)|^{2} d \varrho \leqslant c_{\gamma} \int_{0}^{2 a} \varrho^{2 \gamma}|g(\varrho)|^{2} d \varrho
$$

segue l'asserto.

LeMma 1.2. - Se riesce $\beta<-\frac{1}{2}$ e $g(0)=0$ si ha:

$$
\int_{0}^{\infty} \varrho^{2 \beta}|g(\varrho)|^{2} d \varrho \leqslant c \int_{0}^{\infty} \varrho^{2(\rho+1)}\left|g^{\prime}(\varrho)\right|^{2} d \varrho
$$

per tutte le funzioni g per eui hanno senso tali integrali.

Poichè :

$$
\int_{0}^{\infty} \varrho^{2 \beta}|g(\varrho)|^{2} d \varrho=\int_{0}^{\infty} \varrho^{2 \beta}\left|\int_{0}^{\varrho} g^{\prime}(t) d t\right|^{2} d \varrho
$$

applicando il Lemma 2.1, pag. 286 di [6] si ha l'asserto.

Lemma 1.3. - Se $a \in R_{+}, \lambda_{0} \in \bar{R}_{+}, \alpha<\frac{1}{2}$ e $\gamma \in R \exists$ e dipendente $d a \lambda_{0}$ tale che per $0 \leqslant \lambda \leqslant \lambda_{0}$ riesce

$$
|g(\lambda)|^{2} \leqslant c\left(\int_{\lambda}^{\lambda+a} \varrho^{2 \alpha}\left|g^{\prime}(\varrho)\right|^{2} d \varrho+\int_{\lambda}^{\lambda+a}|g(\varrho)|^{2} d \varrho\right) \leqslant c_{\gamma}\left(\int_{\lambda}^{\lambda+2 a} \varrho^{2 \alpha}\left|g^{\prime}(\varrho)\right|^{2} d \varrho+\int_{\lambda}^{\lambda+2 a} \varrho^{2 \gamma}|g(\varrho)|^{2} d \varrho\right)
$$

per tutte le funzioni g per oui hanno senso tali integrali. 
Osserviamo che:

$$
|g(\lambda)|^{2}=\left|-\int_{\lambda}^{\varrho} g^{\prime}(t) d t+g(\varrho)\right|^{2} \leqslant 2|g(\varrho)|^{2}+2\left|\int_{\lambda}^{\varrho} g^{\prime}(t) d t\right|^{2} .
$$

Applicando la diseguaglianza di Schwartz-Holder si ricava:

$$
\left|\int_{\lambda}^{e} g^{\prime}(t) d t\right|^{2} \leqslant \int_{\lambda}^{\varrho} t-2 \alpha d t \cdot \int_{\lambda}^{\varrho} t^{2 \alpha}\left|g^{\prime}(t)\right|^{2} d t=\frac{Q^{1-2 \alpha}-\lambda^{1-2 \alpha}}{1-2 \alpha} \int_{\lambda}^{\varrho} t t^{2 \alpha}\left|g^{\prime}(t)\right|^{2} d t \leqslant \frac{Q^{1-2 \alpha}}{1-2 \alpha} \int_{\lambda}^{\varrho} t^{2 \alpha}\left|g^{\prime}(t)\right|^{2} d t
$$

se $g \geq \lambda$.

Quindi:

$$
|g(\lambda)|^{2} \leqslant 2|g(\varrho)|^{2}+\frac{2}{1-2 \alpha} \cdot \varrho^{1-2 \alpha} \int_{\lambda}^{\varrho} t^{2 \alpha}\left|g^{\prime}(t)\right|^{2} d t
$$

Integrando tra $\lambda$ e $\lambda+a$ in $d \varrho$ ed osservando che per la formula di Dirichlet si ha:

$$
\int_{\lambda}^{\lambda+a}\left[\varrho^{1-2 \alpha} \int_{\lambda}^{\varrho} t^{2 \alpha}\left|g^{\prime}(t)\right|^{2} d t\right] d \varrho=\int_{\lambda}^{\lambda+a}\left(\int_{i}^{\lambda+a} t^{2 \alpha}\left|g^{\prime}(t)\right|^{2} \varrho^{1-2 \alpha} d \varrho\right) d t \leqslant \int_{\lambda}^{\lambda+a} \varrho^{1-2 \alpha} \int_{\lambda}^{\lambda+a} t^{2 \alpha}\left|g^{\prime}(t)\right|^{2} d t \leqslant c \int_{\lambda}^{\lambda+a} t^{2 \alpha}\left|g^{\prime}(t)\right|^{2} d t
$$

segue l'asserto.

La seconda parte della (1.2) è banale se $\lambda>0$; segue dalla (1.1) se $\lambda=0$.

Lemma 1.4. - Se $a \in R_{+}, \gamma \in R, \beta>-1$ vale:

$$
\begin{aligned}
\int_{A_{a}}|x|^{2 \beta}|u|^{2} d x \leqslant e\left(\int_{\Lambda_{z a}}|x|^{2(\beta+1)}|\partial u|^{2} d x+\int_{A_{z a}-\Lambda_{a}}|u|^{2} d x\right) & =e_{\nu}\left(\int_{\Lambda_{2 a}}|x|^{2(\beta+1)}|\partial u|^{2} d x+\int_{A_{z a}}|x|^{2 \gamma}|u|^{2} d x\right)
\end{aligned}
$$

per tutte le funzioni u per cui hanno senso tali integrali.

Riesce:

$$
\int_{A_{a}}|x|^{2 \beta}|u|^{2} d x=\int_{\theta_{1}}^{\theta_{2}} d \theta \int_{0}^{a} \varrho^{2 \beta+1}\left|u_{\pi}(\varrho, \theta)\right|^{2} d \varrho
$$

Applicando la (1.1) si ricava:

$$
\int_{A_{a}}|x|^{2 \beta}|u|^{2} d x \leqslant c\left(\int_{\theta_{1}}^{\theta_{2}} d \theta \int_{0}^{2 a} \varrho^{2 \beta+3}\left|\partial_{\varrho} u_{\pi}(\varrho, \theta)\right|^{2} d \rho+\int_{\theta_{1}}^{\theta_{3}} d \theta \int_{a}^{2 a}\left|u_{\pi}(\varrho, \theta)\right|^{2} d \varrho\right) .
$$


Dal fatto che:

$$
\partial_{\ell} u_{\pi}(\varrho, \theta)=\left(\partial_{x_{1}} u \cdot \cos \theta+\partial_{x_{2}} u \cdot \operatorname{sen} \theta\right)_{\pi}
$$

segue l'asserto.

LEMMA 1.5. $-\$ s \beta<-1$ e $u(0)=0$ vale:

$$
\int_{A}|x|^{2 \beta}|u|^{2} d x \leqslant c \int_{A}|x|^{2(\beta+1)}|\partial u|^{2} d x
$$

per le funzioni per cui hanno senso tali integrali.

Osservando che:

$$
\int_{A}|\alpha|^{\prime 2 \beta}|u|^{2} d x=\int_{\theta_{1}}^{\theta_{2}} d \theta \int_{0}^{\infty} \varrho^{2 \beta+1}\left|u_{\pi}(\varrho, \theta)\right|^{2} d \varrho
$$

ed applicando la $\left(1.1^{\prime}\right)$ segue l'asserto.

Lemma 1.6. - $\forall \alpha \in R, a \in R_{+}, \theta_{0} \in\left[\theta_{1}, \theta_{2}\right]$ vale la disuguaglianza:

$$
\int_{\Lambda_{a}}|x|^{2 \alpha}|u|^{2} d x \leqslant c\left(\int_{\Lambda_{a}}|x|^{2(\alpha+1)}|\partial u|^{2} d x+\int_{0}^{a} \varrho^{2 \alpha+1}\left|u_{\pi}\left(\varrho, \theta_{0}\right)\right|^{2} d \varrho\right)
$$

per le funzioni per oui hanno senso tali integrali.

Si ha:

$$
\left|u_{\pi}(\varrho, \theta)\right|^{2}=\left|\int_{\theta_{0}}^{\theta} \partial_{\tau} u_{\pi}(\varrho, \tau) d \tau+u_{\pi}\left(\varrho, \theta_{0}\right)\right|^{2} \leqslant 0\left[\int_{\theta_{1}}^{\theta_{2}}\left|\partial_{\tau} u_{n}(\varrho, \tau)\right|^{2} d \tau+\left|u_{n}\left(\varrho, \theta_{0}\right)\right|^{2}\right]
$$

tenuto conto che:

$$
\partial_{\theta} u(\varrho \cos \theta, \varrho \operatorname{sen} \theta)=\left(-\varrho \operatorname{sen} \theta \partial_{x_{2}} u+\varrho \cos \theta \partial_{x_{2}} u\right)_{\pi}
$$

si ha:

$$
\left|u_{z}(\varrho, \theta)\right|^{2} \leqslant c\left(\int_{\theta_{1}}^{\theta_{3}} \varrho^{2}\left|(\partial u)_{\pi}\right|^{2} d \tau+\left|u_{\pi}\left(\varrho, \theta_{0}\right)\right|^{2}\right)
$$

Moltiplicando per $\varrho^{2 \alpha+1}$ ambo i membri ed integrando tra 0 ed $a$ in $d \varrho$ e poi tra $\theta_{1}$ e $\theta_{2}$ in $d \theta$ segue la (1.4).

LEMMA 1.7. $-\forall \alpha \in R, \forall a \in R_{+}, \forall \varepsilon \in R_{+}$, riesce con $\theta_{0} \in\left[\theta_{1}, \theta_{2}\right]$ :

$$
\int_{0}^{a} \varrho^{2 \alpha+1}\left|u_{n}\left(\varrho, \theta_{0}\right)\right|^{2} d \varrho \leqslant \varepsilon \int_{A_{a}}|x|^{2(\alpha+1)}|\partial u|^{2} d x+c(\varepsilon) \int_{A_{a}}|x|^{2 \alpha}|u|^{2} d x
$$


se $\alpha \in]-1,+\infty[$ e $\gamma \in R$ si ha:

$$
\int_{0}^{a} \varrho^{2 \alpha+1}\left|u_{\pi}\left(\varrho, \theta_{0}\right)\right|^{2} \leqslant c_{\nu}\left(\int_{\Lambda_{z a}}|x|^{2(\alpha+1)}|\partial u|^{2} d x+\int_{A_{z a}}|x|^{2 \gamma}|u|^{2} d x\right)
$$

per le funzioni per cui hanno senso tali integrali.

Si osservi che:

$$
z \bar{w}+w \bar{z}=2 \operatorname{Re}(\bar{z} \cdot w) \leqslant 2|z| \cdot|w| \leqslant \varepsilon^{2}|z|^{2}+\frac{1}{\varepsilon^{2}}|w|^{2} .
$$

Quindi:

$$
\partial_{\theta}\left|u_{\pi}\right|^{2}=\partial_{\theta}\left(u_{\pi} \cdot \bar{u}_{\pi}\right) \leqslant \varepsilon^{2}\left|\partial_{\theta} u_{\pi}\right|^{2}+\frac{1}{\varepsilon^{2}}\left|u_{\pi}\right|^{2}
$$

Da qui:

$$
\begin{aligned}
\left|u_{\pi}\left(\varrho, \theta_{0}\right)\right|^{2}=-\int_{\theta_{0}}^{\theta} \partial_{\tau}\left|u_{\pi}(\varrho, \tau)\right|^{2} d \tau+\left|u_{\pi}(\varrho, \theta)\right|^{2} & \\
& \leqslant \varepsilon^{2} \int_{\theta_{1}}^{\theta_{2}}\left|\partial_{\tau} u_{\pi}\right|^{2} d \tau+\frac{1}{\varepsilon^{2}} \int_{\theta_{1}}^{\theta_{3}}\left|u_{\pi}\right|^{2} d \tau+\left|u_{\pi}(\varrho, \theta)\right|^{2} \\
& \leqslant 2 \varepsilon^{2} \int_{\theta_{1}}^{\theta_{2}} \varrho^{2}\left(\left|\left(\partial_{x_{1}} u\right)_{\pi}\right|^{2}+\left|\left(\partial_{x_{3}} u\right)_{\pi}\right|^{2}\right) d \tau+\frac{1}{\varepsilon^{2}} \int_{\theta_{1}}^{\theta_{3}}\left|u_{\pi}\right|^{2} d \tau+\left|u_{\pi}(\varrho, \theta)\right|^{2} .
\end{aligned}
$$

Moltiplicando per $\varrho^{2 \alpha+1}$, integrando tra 0 ed $a$ in $d \varrho$ e poi tra $\theta_{1}$ e $\theta_{2}$ in $d \theta$ segue la (1.5).

La $\left(1.5^{\prime}\right)$ segue immediatamente dalla (1.3) e (1.5).

Lemna 1.8. - Se $\alpha \in]-\infty, \frac{1}{2}\left[, a \in R_{+}\right.$, vale la seguente diseguaglianza:

$$
\left|u\left(t_{1}, t_{2}\right)\right|^{2} \leqslant c \sum_{|\beta| \leqslant 2} \int_{A_{2}(\sigma+a)}|x|^{2\left(\alpha+\frac{1}{2}\right)} \mid \partial^{\beta} u^{2} d x
$$

con $\sigma=\sqrt{t_{1}^{2}+t_{2}^{2}} \leqslant \sigma_{0} \in \bar{R}_{+}$e o dipendente da $\sigma_{0}$ e per tutte le funzioni per cui abbiano senso tali integrali.

Basta provare la (1.6) per $\alpha \in]-\frac{1}{2}, \frac{1}{2}[$.

Dalla (1.2) segue:

$$
\begin{aligned}
\left|u\left(t_{1}, t_{2}\right)\right|^{2}=\left|u_{\pi}\left(\sigma, \theta_{0}\right)\right|^{2} \leqslant c\left(\int_{\sigma}^{\sigma+a} \varrho^{2 \alpha}\left|\partial_{\varrho} u_{\pi}\left(\varrho, \theta_{0}\right)\right|^{2} d \varrho\right. & \left.+\int_{\sigma}^{\sigma+a} \varrho^{2 \alpha} \mid u_{\pi}\left(\varrho, \theta_{0}\right)^{2} d \varrho\right) \leqslant \\
& \leqslant c\left(\int_{0}^{\sigma+a} \varrho^{2 \alpha}\left|\partial_{\varrho} u_{\pi}\left(\varrho, \theta_{0}\right)\right|^{2} d \varrho+\int_{0}^{\sigma+a} \varrho^{2 \alpha}\left|u_{\pi}\left(\varrho, \theta_{0}\right)\right|^{2} d \varrho\right) .
\end{aligned}
$$


Applicando, allora, la $\left(1.5^{\prime}\right)$ si ottiene:

$$
\left|u\left(t_{1}, t_{2}\right)\right|^{2} \leqslant c\left(\int_{A_{2}(\sigma+a)}|x|^{2\left(\alpha+\frac{1}{2}\right)}\left|\partial^{2} u\right|^{2} d x+\int_{A_{2}(\sigma+a)}|x|^{2\left(\alpha+\frac{1}{2}\right)}|\partial u|^{2} d x+\int_{A_{2}(\sigma+\alpha)}|x|^{2\left(\alpha+\frac{1}{2}\right)}|u|^{2} d x\right)
$$

quindi la (1.6).

Lemma 1.9. - Se $s<1$ vale $\forall \varepsilon \in] 0,1]$ con $a \in R_{+}$:

$$
|u(0,0)|^{2} \leqslant \varepsilon\left(\int_{\Lambda_{a}}|x|^{2 s}\left|\partial^{2} u\right|^{2} d x+\int_{\Lambda_{a}}|x|^{2(s-1)}|\partial u|^{2} d x\right)+c(\varepsilon) \int_{A_{a}}|x|^{2 s}|u|^{2} d x
$$

Posto $\frac{1}{2}+\alpha=s$, dalla (1.6) con $t_{1}=t_{2}=0$ si ricava con $v(x)=u(\varepsilon x)$ :

$$
|v(0,0)| \leqslant c\left(\int_{A_{a}}|x|^{2 s}\left|\partial^{2} v\right|^{2} d x+\int_{A_{x}}|x|^{2(s-1)}|\partial v|^{2} d x+\int_{A_{a}}|x|^{2 s}|v|^{2} d x\right)
$$

Posto:

$$
\varepsilon x=\left(\varepsilon x_{1}, \varepsilon x_{2}\right)=\left(t_{1}, t_{2}\right)=t
$$

si ricava:

$$
|u(0,0)|^{2} \leqslant o\left(\int_{|t| \leqslant \varepsilon a}\left|\frac{t}{\varepsilon}\right|^{2 s} \cdot \varepsilon^{4}\left|\partial^{2} u\right| \varepsilon^{-2} d t+\int_{|t| \leqslant \varepsilon a}\left|\frac{t}{\varepsilon}\right|^{2(\delta-1)} \varepsilon^{2}|\partial \hat{\partial} u|^{2} \varepsilon^{-2} d t+\int_{|t| \leqslant \varepsilon a}\left|\frac{t}{\varepsilon}\right|^{2 s}|u|^{2} \varepsilon^{-2} d t\right)
$$

quindi la (1.7).

2. - Gli spazi $U_{s_{1}, s_{3}}^{r}(\Lambda)$ e $V_{s_{1}, s_{2}}^{r}(\Lambda)$.

Sia $r$ un intero $\geqslant 2$ ed $s_{1}, s_{2}$ due numeri reali con $s_{1} \leqslant s_{2} ;$ poniamo per definizione $\left(^{(1)}\right.$ :

$$
\begin{aligned}
\|u\|_{U_{s_{1}, s_{1}}^{r}(A)}^{2}=\sum_{|\alpha| \leqslant r-2} \int_{A_{2}}\left|\partial^{\alpha} u\right|^{2} d x & +\sum_{|\alpha|=r-1} \sum_{|\beta| \leqslant 1} \int_{A_{2}}|x|^{2\left\langle s_{1}+|\beta|-1\right)}\left|\partial^{\alpha+\beta} u\right|^{2} d x+ \\
& +\left.\sum_{|\alpha| \leqslant r}|| x\right|^{2\left(s_{2}+|\alpha|-r\right)}\left|\partial^{\alpha} u\right|^{2} d x . A_{1}
\end{aligned}
$$

Indicheremo con $U_{s_{1}, s_{3}}^{r}(\Lambda)$ lo spazio delle funzioni $u(x)$ tali che la norma (2.1) sia finita se $r \geqslant 2$, lo spazio $W_{s_{1}, s_{2}}^{r}(\Lambda)$ (cfr. [1]) se $r<2$.

Supporremo da ora in poi, salvo esplicito avviso contrario, $r \geqslant 2$.

Indicheremo talvolta la seconda e terza sommatoria a destra della (2.1) rispettivamente con:

$$
\sum_{|\alpha|=r-1}\left\|\partial^{\alpha} u\right\|_{W_{s_{k}}^{1}\left(A_{2}\right)}^{2},\|u\|_{W_{s_{2}}^{r}\left(\Lambda-A_{1}\right)}^{2}
$$

$\left.{ }^{1}\right)$ Con $\partial^{\alpha} u$ si intendono le derivate di $u$ nel senso delle distribuzioni. 
Detto $r$ un intero $\geqslant 0$ indicheremo con $V_{s_{1}, s_{2}}^{*}(\Lambda)$ lo spazio delle funzioni $u(x)$ tali che sia finita la norma:

$$
\begin{aligned}
& \|u\|_{V_{s_{1}, s_{2}}^{r}(\Lambda)}^{2}=\sum_{|\alpha| \leqslant r} \int_{\Lambda_{\mathrm{a}}}|x|^{2 s_{1}}\left|\partial^{\alpha} u\right|^{2} d x+ \\
& +\sum_{|\alpha| \leqslant r} \int_{A-A_{1}}|x|^{2\left(s_{2}+|\alpha|-r\right)}\left|\partial^{\alpha} u\right|^{2} d x=\|u\|_{W_{2,|x|^{r}}^{2} s_{1}\left(\Lambda_{2}\right)}^{2}+\|u\|_{W_{s_{2}}^{r}\left(A-A_{1}\right)}^{2},
\end{aligned}
$$

(per la definizione di $W_{2,|x|^{2 s_{1}}}^{r}\left(\Lambda_{2}\right)$ efr. [6], cap. 6).

Valgono i seguenti lemmi di inclusione:

LEMMMa 2.1. $-S e s_{1} \leqslant 2 \leqslant r$

$$
W_{s_{1}, s_{2}}^{r}(A) \subset U_{s_{1} s_{2}}^{r}(\Lambda)
$$

algebricamente e topologicamente (1).

Basta osservare che:

$$
s_{1}+|\alpha|-r \leqslant 0 \quad \text { per }|\alpha| \leqslant r-2 .
$$

LEMIMA 2.2. $-\forall s_{1} \geqslant 0$ si ha:

$$
\left.U_{s_{1}, s_{z}}^{r}(\Lambda) \subset V_{s_{1}, s_{z}}^{r} A\right)
$$

algebricamente e topologicamente.

La dimostrazione è evidente.

LENMLA 2.3. - Se riesce $0<s_{1} \leqslant 1$ si ha:

$$
U_{s_{1}, \delta_{2}}^{*}(\Lambda) \simeq V_{s_{1}, s_{8}}^{*}(\Lambda)
$$

intendendo con tale simbolo che tali spazi sono isomorfi algebricamente e topologicamente.

In virtù del Lemma 2.2 basterà dimostrare che riesce:

$$
V_{s_{1}, s_{2}}^{r}(\Lambda) \subset U_{s_{1}, s_{3}}^{r}(\Lambda)
$$

per la (1.3) si ha:

$$
\begin{aligned}
\sum_{\mid \alpha ; \leqslant r-2} \int_{A_{1}}\left|\partial^{\alpha} u\right|^{2} d x \leqslant c\left(\sum_{|\alpha| \leqslant r-1} \int_{A_{2}}|x|^{2}\left|\partial^{\alpha} u\right|^{2} d x+\right. & \left.\sum_{|\alpha| \leqslant r-2} \int_{A_{3}}|x|^{2}\left|\partial^{\alpha} u\right|^{2} d x\right) \leqslant \\
& \leqslant c^{\prime}\left(\sum_{|\alpha| \leqslant r-1} \int_{A_{2}}|x|^{2 s_{1}}\left|\partial^{\alpha} u\right|^{2} d x\right) \leqslant c^{\prime \prime}\|\| u \|_{V_{s_{1}, s_{2}}^{*}(\Lambda)}^{2} .
\end{aligned}
$$

D'altro canto riesce:

$$
\sum_{|\alpha|=r-1}\left\|\partial^{\alpha} u\right\|_{W_{s_{1}}^{1}\left(A_{2}\right)}^{2} \leqslant c\|u\|_{S_{s_{1}, s_{2}}^{r}(A)}^{2},
$$

(1) Ricordiamo che: $\|u\|_{\mathbb{S}_{s_{1}, s_{2}}^{r}(A)}^{2}=\sum_{|\alpha| \leqslant r}\left(\int_{A}|x|^{2\left(s_{1}+|\alpha|-r\right)}\left|\partial^{\alpha} u\right|^{2} d x+\int_{A}|x|^{2\left(s_{2}+|\alpha|-r\right) \mid}\left|\hat{\partial}^{\alpha} u\right|^{2} d x\right)$. 
poichè per la (1.3) si ha:

$$
\int_{\Lambda_{1}}|x|^{2\left(\delta_{1}-1\right)}\left|\partial^{r-1} u\right|^{2} d x \leqslant c\left(\int_{\Lambda_{2}}|x|^{2 s_{1}}\left|\partial^{r} u\right|^{2} d x+\int_{A_{2}}|x|^{2 s_{2}}\left|\partial^{r-1} u\right|^{2} d x\right) .
$$

In definitiva, quindi:

$$
\|u\|_{U_{s_{1}, s_{2}}^{r}(\Lambda)} \leqslant \theta\|u\|_{\Gamma_{s_{1}, s_{2}}^{r}(\Lambda)}
$$

cioè l'asserto.

Lemia 2.4. - Se $s_{1} \geqslant 0, \mathfrak{D}(\bar{\Lambda}) \grave{e}$ denso in $V_{s_{1} . s_{2}}^{r}(\Lambda)$.

Sia $u \in V_{s_{k}, s_{\mathrm{g}}}^{r}(\Lambda)$.

Siano $\zeta_{1}(x)$ e $\zeta_{2}(x)$ due funzioni di classe $C^{\infty}(\bar{\Lambda})$ tali che:

$$
\begin{array}{ll}
\zeta_{1}(x)=0 & \text { per }|x| \geqslant \frac{3}{2}, \\
\zeta_{2}(x)=0 & \text { per }|x| \leqslant 1, \\
\zeta_{1}(x)+\zeta_{2}(x)=1 & \text { in } \bar{A} .
\end{array}
$$

Si ha:

$$
\zeta_{1} u \in W_{2,\left.|x|\right|^{s_{1}}}^{r}\left(\Lambda_{2}\right), \quad \zeta_{2} u \in W_{s_{1}, s_{2}}^{r}(\Lambda)
$$

Per il Teor. 2.1, pag. 284 di [6] esiste una successione $v_{n} \in \mathfrak{D}\left(\bar{\Lambda}_{2}\right)$ che converge a $\zeta_{1} u$ in $W_{2,|x|^{2 s_{1}}}^{r}\left(\Lambda_{2}\right)$.

Sia $u_{n}^{1}$ il prolungamento di $v_{n}$ con valori nulli $|x| \geqslant 2$.

Analogamente esiste per il Teor. 8.1 di [1] una successione di funzioni $u_{n}^{2} \in$ $\in \mathfrak{D}(\bar{\Lambda}-\{0\})$, nulle in un intorno dell'origine, che converge a $\zeta_{2} u$ in $W_{s_{1}, s_{2}}^{r}(\Lambda)$.

Posto:

$$
u_{n}=u_{n}^{1}+u_{n}^{2}
$$

si ha:

$$
\left\|u-u_{n}\right\|_{Y_{s_{1}, s_{2}}^{*}(\Lambda)} \leqslant c\left(\left\|u_{n}^{1}-\zeta_{1} u\right\|_{W_{2, \mid x]^{2} s_{1}\left(A_{3}\right)}}+\left\|u-\zeta_{2} u\right\|_{W W_{s_{2}, s_{2}}^{*}(\Lambda)}\right)
$$

e facendo divergere $n$ segue l'asserto.

Poniamo:

$$
\left[s_{1}\right]=\left\{\begin{array}{l}
\text { al più grande intero minore od uguale ad } s_{1} \text { se } s_{1} \geqslant 0, \\
0 \text { se } s_{1}<0 .
\end{array}\right.
$$

TEOREMA 2.1. - Assegnati $r, s_{1}, s_{2}$ sussiste l'inclusione algebrica e topologica:

$$
U_{s_{1}, s_{2}}^{*}(A) \subset C^{v}\left(\bar{A}_{a}\right) \quad \forall a \in R_{+}
$$


dove si è posto:

$$
\begin{array}{ll}
v=r-2-\left[s_{1}\right] & \text { per } s_{1}<2 \text { ed } r \geqslant 2+\left[s_{1}\right], \\
v=r-4 & \text { per } s_{1} \geqslant 2 \text { ed } r \geqslant 4 .
\end{array}
$$

Se $s_{1} \leqslant 0$ 1'asserto segue banalmente da noti risultati di Sobolev, quando si osservi che:

$$
U_{s_{1}, s_{2}}^{r}(A) \subset W^{r}\left(A_{a}\right)
$$

Sia $\left.s_{1} \in\right] 0,1[$; in virtù de Lemmi 2.3 e 2.4 basterà dimostrare che:

$$
\sum_{|\alpha| \leqslant r-2} \max _{\bar{A}_{a}}\left|\partial^{\alpha} u(x)\right| \leqslant c\|u\|_{U_{s_{1}, s_{2}}^{r}(A)}, \quad \forall u \in \mathbb{D}(\bar{A})
$$

Dalla (1.6) segue che essendo $\sigma \leqslant a$ :

$$
\left|\partial^{\alpha} u(x)\right|^{2} \leqslant c \sum_{|\beta| \leqslant 2} \int_{A_{4^{a}}}|x|^{2 \varepsilon_{1}}\left|\partial^{\alpha+\beta} u\right|^{2} d x \leqslant c^{t}\|u\|_{U_{s_{1}, \varepsilon_{2}}^{r}}
$$

Sia $s_{1} \in[1,2[$; riesce $v=r-3$.

Si ha facilmente che:

$$
U_{s_{1}, s_{a}}^{r}(\Lambda) \subset W_{2,\left.j x\right|^{2\left(s_{1}-1\right)}}^{r-1}\left(\Lambda_{a}\right)
$$

dove $W_{2,|x|^{2\left(\beta_{1}-1\right)}}^{r-1}\left(A_{a}\right)$ denotano gli spazi definiti al Cap. 6 di [6].

D'altro canto essendo $s_{1}-1 \geqslant 0$, per il Teor. 2.1, pag. 284 di $[6], \mathfrak{D}\left(\bar{A}_{a}\right)$ è denso su $W_{2, \mid x)^{2\left(\varepsilon_{q}-1\right)}}^{r-1}\left(A_{a}\right)$.

Applicando la (1.6) si ha allora per $|\alpha| \leqslant r-3$ :

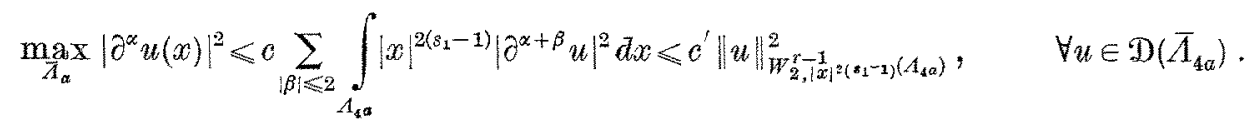

Se $s_{1} \in[2,+\infty[$ l'asserto segue banalmente dal fatto che:

$$
U_{s_{1}, s_{2}}^{r}(A) \subset W^{\varphi-2}\left(\Lambda_{a}\right)
$$

e dal teorema di Sobolev.

\section{3. - Gli spazi $\stackrel{\circ}{U}_{s_{1}, s_{2}}^{r}(\Lambda)$.}

Si pone per definizione:

$$
U_{s_{1}, s_{2}}^{r}(\Lambda)=\left\{u \in U_{s_{1}, s_{2}}^{r}(\Lambda): \partial^{\gamma} u(0,0)=0 \text { per }|\gamma| \leqslant v\right\}
$$


$\operatorname{con}$

$$
v \begin{cases}=r-2-\left[s_{1}\right] & \text { se } s_{1}<2 \\ =r-4 & \text { se } s_{1} \geqslant 2\end{cases}
$$

Vale il seguente

LEMIMA 3.1. - Se $s_{1}<2 \leqslant r, s_{1}+1 \leqslant r e s_{1} \neq 1$

$$
\dot{U}_{s_{1}, s_{2}}^{r}(\Lambda) \simeq W_{s_{1}, s_{2}}^{r}(\Lambda)
$$

In virtù del Lemma 2.1 e della proposizione 9.1 di [1] dobbiamo solo dimostrare che:

$$
\mathscr{U}_{s_{1}, s_{2}}^{r}(\Lambda) \subset W_{s_{1}, s_{2}}^{r}(\Lambda)
$$

Osserviamo intanto che:

$$
\|u\|_{W_{s_{1}, s_{g}}^{r}(\Lambda)}^{2} \leqslant 2 \sum_{|\alpha| \leqslant r} \int_{\Lambda_{1}}|x|^{2\left(s_{1}+|\alpha|-x\right)}\left|\partial^{\alpha} u\right|^{2} d x+2\|u\|_{W_{s_{2}}^{r}\left(\Lambda-A_{1}\right)}^{2} .
$$

Si ha intanto $\forall s_{1}$

$$
\sum_{|\alpha|=r+1} \int_{A_{1}}|x|^{2\left(s_{1}-1\right)}\left|\partial^{\alpha} u\right|^{2} d x+\sum_{|\alpha|=r} \int_{\Lambda_{1}}|x|^{2 s_{1}}\left|\partial^{\alpha} u\right|^{2} d x \leqslant \sum_{|\alpha|=r-1}\left\|\partial^{\alpha} u\right\|_{W_{s_{1}}^{1}\left(A_{3}\right)}^{2} .
$$

Sia $s_{1}<1$; restano da maggiorare i termini del tipo:

$$
\int_{\mathcal{A}_{1}}|x|^{2\left(s_{1}-k\right)}\left|\hat{o}^{\alpha} u\right|^{2} d x
$$

con $|\alpha|=r-k$ e $k-2 \geqslant 0$.

Poichè $s_{1}-k<-1$, per il teorema 2.1 e applicando la $\left(1.3^{\prime}\right) k-1$ volte si ottiene:

$$
\begin{aligned}
& \int_{\Lambda_{1}}|x|^{2\left(s_{1}-k\right)}\left|\partial^{\alpha} u\right|^{2} d x \leqslant c\left(\int_{\Lambda_{1}}|x|^{2\left(s_{1}-1\right)}\left|\partial^{r-1} u\right|^{2} d x+\int|x|^{2\left(s_{2}-1\right)}\left|\partial^{r-1} u\right|^{2} d x \leqslant:\right. \\
& \quad \leqslant c\left(\sum_{|\alpha|=r-1}\left\|\partial^{\alpha} u\right\|_{W_{s_{1}}^{\mathbf{1}}\left(\Lambda_{2}\right)}^{2}+\|u\|_{W_{s_{2}}^{r}\left(A-A_{1}\right)}^{2}\right)
\end{aligned}
$$

Se poi $\left.s_{1} \in\right] 1,2[$ si applica la (1.3) alle derivate di ordine $r-2$ e poi si procede come sopra. 
4. - Spazi $U_{s_{1} \frac{1}{2}, s_{2}+\frac{1}{2}}^{r-\frac{1}{2}}\left(R_{+}\right), U_{s_{1}, s_{2}}^{r-\frac{1}{2}}\left(\sigma_{k}\right), \dot{U}_{s_{1}+\frac{1}{2}, s_{2}+\frac{1}{2}}^{r-\frac{1}{2}}\left(R_{+}\right)$.

Assegnati $s_{1} \leqslant s_{2}$ in $R$ ed $r$ intero positivo, indicheremo con $U_{s_{+}+\frac{1}{2}, s_{2}+\frac{1}{2}}^{r-\frac{1}{2}}\left(R_{+}\right)$lo spazio delle funzioni $g(\varrho)$ tali che sia finita la norma:

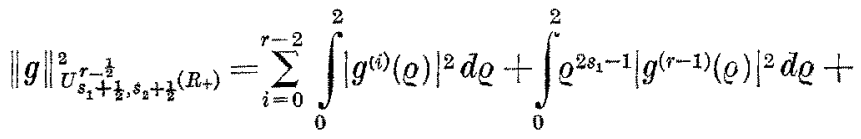

$$
\begin{aligned}
& +\int_{-\infty}^{\log 2} \int_{-\infty}^{\log 2} \frac{\left|e^{s_{2} y} g^{(r-1)}\left(e^{y}\right)-e^{s_{1} y^{\prime}} g^{(r-1)}\left(e^{y^{\prime}}\right)\right|^{2}}{\left|y-y^{\prime}\right|^{2}} d y d y^{\prime}+\sum_{i=0}^{r-1} \int_{1}^{+\infty} e^{2\left(s_{2}+i-1\right)+1}\left|g^{(i)}(\varrho)\right|^{2} d \varrho \\
& +\int_{0}^{+\infty} \int_{0}^{+\infty} \frac{\left|e^{\delta_{2} y} g^{(r-1)}\left(e^{y}\right)-e^{s_{2} y^{\prime}} g^{(r-1)}\left(e^{y^{\prime}}\right)\right|^{2}}{\left|y-y^{\prime}\right|^{2}} d y d y^{\prime}
\end{aligned}
$$

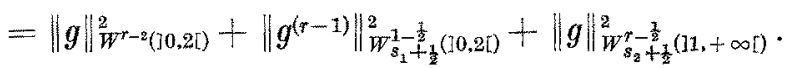

se $r \geqslant 2 ;$ lo spazio $W_{s_{1}+\frac{1}{2}, s_{2}+\frac{1}{2}}^{r-\frac{1}{2}}\left(R_{+}\right)$se $r<2\left({ }^{1}\right)$.

Indicheremo, inoltre, con $V_{s_{1}, s_{2}}^{r-\frac{1}{2}}\left(\sigma_{k}\right)$ lo spazio della funzioni definite su $\sigma_{k}$ tali che:

$$
g_{k}(\varrho)=g\left(\varrho \cos \theta_{k}, \varrho \operatorname{sen} \theta_{k}\right) \in U_{s_{1}+\frac{1}{2}, s_{2}+\frac{1}{2}}^{r-\frac{1}{2}}\left(R_{+}\right) .
$$

Vale il seguente:

Lemina 4.1. - Se $s_{1} \leqslant \frac{3}{2}$ si ha:

$$
W_{s_{1}+\frac{1}{2}, s_{2}+\frac{1}{3}}^{r-\frac{1}{3}}\left(R_{+}\right) \subset U_{s_{1}+\frac{1}{2}, s_{2}+\frac{1}{2}}^{r-\frac{1}{2}}\left(R_{+}\right)
$$

algebricamente e topologicamente.

Osserviamo che da $s_{1}+i-r+\frac{1}{2} \leqslant 0$ segue:

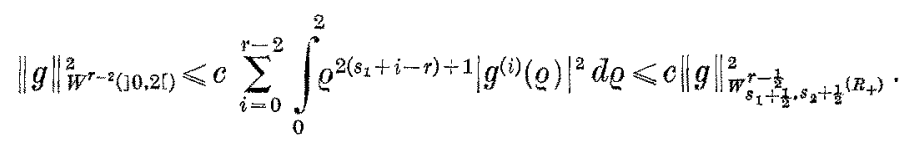

È d'altro canto immediato che:

$$
\begin{aligned}
& \left\|g^{(r-1)}\right\|_{W_{s_{1}+\frac{2}{2}}^{1-\frac{1}{2}(10,2)}}^{2} \leqslant c \| g_{W_{s_{1}+\frac{1}{2}, s_{2}+\frac{1}{2}\left(R_{+}\right)}^{2}}^{2} \\
& \|g\|_{W_{s_{2}+\frac{1}{2}}^{r-\frac{1}{2}(11,+\infty}}^{2} \leqslant \mathcal{L}\|g\|_{W_{s_{1}+\frac{1}{2}, s_{2}+\frac{1}{2}}^{r-\frac{1}{2}}\left(R_{+}\right)}^{2} .
\end{aligned}
$$

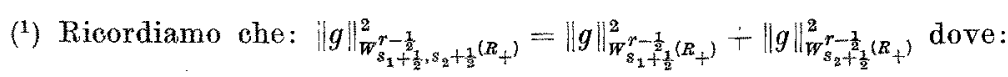

$$
\|g\|_{W_{s+\frac{1}{2}\left(R_{+}\right)}^{r+\frac{1}{2}}}=\sum_{i=0}^{r-1} \int_{R_{+}} \varrho^{2\left(s+i-r+\frac{1}{2}\right)}\left|g^{(i)}(\varrho)\right|^{2} d \varrho+\iint_{R} \frac{\left|e^{s y} g^{(r-1)}\left(e^{y}\right)-e^{s y^{\prime}} g^{(r-1)}\left(e^{y^{\prime}}\right)\right|^{2}}{\left|y-y^{\prime}\right|^{2}} d y d y^{\prime} .
$$


TEOREMA 4.1. - Assegnati $r, s_{1}, s_{2}$ sussiste l'inclusione algebrica e topologica:

$$
U_{s_{1}+\frac{1}{2}, s_{2}+\frac{1}{2}}^{r-\frac{1}{2}}\left(R_{+}\right) \subset C^{v}([0, a]), \quad \forall a \in R_{+}
$$

dove si è posto:

$$
v \begin{cases}=r-2 & \text { se } s_{1}<1 \text { ed } r \geqslant 2, \\ =r-3 & \text { se } s_{1} \geqslant 1 \text { ed } r \geqslant 3\end{cases}
$$

Sia $s_{1} \leqslant \frac{1}{2}$. Riesce ovviamente:

$$
U_{\dot{s}_{1}+\frac{1}{2}, s_{2}+\frac{1}{2}}^{r-\frac{1}{2}}\left(R_{+}\right) \subset W^{r-1}(] 0, a[)
$$

e quindi l'asserto segue dal già citato teorema di Sobolev.

Sia, adesso, $\frac{1}{2}<s_{1}<1$.

Riesce, facilmente:

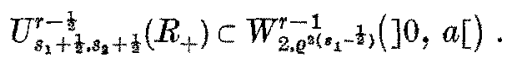

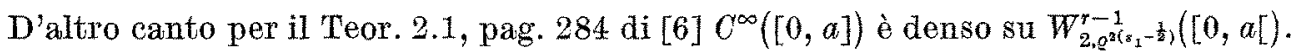
Applicando il Lemma 1.3 si ha:

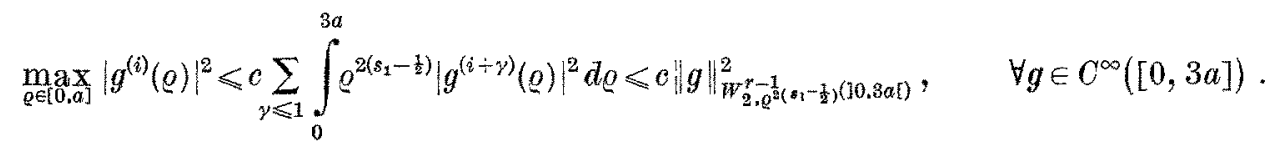

Se $s_{1}>1$ l'asserto segue dal fatto che:

$$
U_{s_{1}+\frac{1}{2}, s_{2}+\frac{1}{2}}^{r-\frac{1}{2}}\left(R_{+}\right) \subset W^{r-2}(] 0, a[) .
$$

Poniamo per definizione:

$$
\mathbb{U}_{s_{1}+\frac{1}{2}, s_{3}+\frac{1}{2}}^{r-\frac{1}{2}}\left(R_{+}\right)=\left\{g \in U_{s_{1}+\frac{1}{2}, s_{2}+\frac{1}{3}}^{r-\frac{1}{3}}\left(R_{+}\right): g^{(i)}(0)=0 \text { per } i \leqslant \nu\right\}
$$

con $v=r-2$ se $s_{1}<1, v=r-3$ se $s_{1} \geqslant 1$.

LEMMA 4.2. - Se riesee $s_{1} \leqslant \frac{3}{2}, s_{1} \neq 1, s_{1}+1 \leqslant r$, si ha:

$$
\stackrel{\circ}{\dot{U}_{s_{1}+\frac{1}{2}, s_{z}+\frac{1}{2}}^{r-\frac{1}{2}}}\left({ }_{+} R\right) \simeq W_{s_{1}+\frac{1}{2}, s_{2}+\frac{1}{2}}^{r-\frac{1}{2}}\left(R_{+}\right) .
$$

In virtù del Lemma 4.1 e della Proposizione 9.1 di [1] basta provare che è:

$$
\stackrel{O}{U}_{\varepsilon_{1}+\frac{1}{2}+s_{2}+\frac{1}{2}}^{r-\frac{1}{2}}\left(R_{+}\right) \subset W_{s_{1}+\frac{1}{2}, s_{2}+\frac{1}{2}}^{r-\frac{1}{2}}\left(R_{+}\right) .
$$


Sia $s_{1}<1$.

$$
\begin{aligned}
& \|g\|_{W_{s_{1}+\frac{1}{2}, s_{\mathrm{s}}+\frac{1}{2}\left(R_{+}\right)}}=\sum_{k=1}^{2} \sum_{i=0}^{r-1} \int_{R_{+}} \varrho^{2\left(s_{k}+i-r\right)+1}\left|g^{(i)}(\varrho)\right|^{2} d \varrho \\
& +\sum_{k=1}^{2} \int_{R} \int_{R} \frac{\left|e^{\delta_{k} y} g^{(r-1)}\left(e^{y}\right)-e^{s k y^{\prime}} g^{(r-1)}\left(e^{v^{\prime}}\right)\right|^{2}}{\left|y-y^{\prime}\right|^{2}} d y d y^{\prime} .
\end{aligned}
$$

Il secondo termine si maggiora procedendo come nel Lemma 9.2 di [1].

Consideriamo $i=(r-1)-k$ con $k \geqslant 1$.

Poichè :

$$
g^{(i)}(0)=0
$$

e poichè:

$$
s_{1}-k-\frac{1}{2}<-\frac{1}{2} \quad \text { per } s_{1}<1
$$

si ha in virtù della (1.1'):

$$
\int_{0}^{1} \varrho^{2\left(s_{1}-k-\frac{1}{2}\right)}\left|g^{(r-1-k)}(\varrho)\right|^{2} d \varrho \leqslant \int_{0}^{+\infty} \varrho^{2 s_{1}-1}\left|g^{(r-1)}(\varrho)\right|^{2} d \varrho,
$$

quindi l'asserto.

Se $\left.s_{1} \in\right] 1, \frac{3}{2}\left[\right.$ si ha $g^{(i)}(0)=0$ per $i \leqslant r-3$.

Consideriamo:

$$
\int_{0}^{1} \varrho^{2\left(s_{1}-\frac{3}{2}\right)}\left|g^{(r-2)}(\varrho)\right|^{2} d \varrho
$$

poichè: $s_{1}-\frac{3}{2}>-\frac{1}{2}$ per $s_{1}>1$ si può applicare la (1.1) e questo termine si maggiora con:

$$
\int_{0}^{2} \varrho^{2\left(s_{x}-\frac{1}{2}\right)}\left|g^{(\gamma-1)}(\varrho)\right|^{2} d \varrho+\int_{0}^{2}\left|g^{(\gamma-2)}(\varrho)\right|^{2} d \varrho
$$

Consideriamo:

$$
i=(r-1)-k, \quad k \geqslant 2 ;
$$

poichè è: $g^{(i)}(0)=0$ e $s_{1}-k-\frac{1}{2}<-\frac{1}{2}$ per $s_{1}<2$ si ha applicando la $\left(1.1^{\prime}\right)$ :

$$
\int_{0}^{1} \varrho^{2\left(s_{1}-k-\frac{1}{2}\right)}\left|g^{(r-1-k)}\right|^{2} d \varrho \leqslant \int_{0}^{+\infty} \varrho^{2\left(s_{1}-\frac{3}{2}\right)}\left|g^{(r-2)}\right|^{2} d \varrho
$$

e da qui l'asserto. 


\section{5. - Teoremi di densità e di tracee.}

Assegnato un intero $v$ consideriamo l'insieme di funzioni $R_{v}(\bar{\Lambda})$ così definito:

$$
u \in R_{v}(\bar{A}) \Leftrightarrow \exists v \in \mathfrak{D}(\bar{\Lambda}-\{0\})
$$

ed esiste un polinomio $Q_{\nu}(x)$ di grado minore od uguale a $v$ tale che sia:

$$
u(x)=v(x)+\chi(x) Q_{p}(x)
$$

dove $\chi(x)$ è un'assegnata funzione di classe $D(\bar{\Lambda})$ tale che riesca:

$$
\chi(x) \begin{cases}=1 & |x| \leqslant 1, \\ =0 & |x| \geqslant 2 .\end{cases}
$$

È ovvio che:

$$
R_{v}(\bar{\Lambda}) \subset \mathfrak{D}(\bar{\Lambda})
$$

e che si ha:

$$
v^{\prime}<v^{\prime \prime} \Rightarrow R_{v^{\prime}}(\bar{\Lambda}) \subset R_{v^{\prime \prime}}(\bar{\Lambda})
$$

Vale il seguente teorema di densità:

TeOReila 5.1. - Se è $s_{1}<2, s_{1}+1 \leqslant r$ ed $s_{1} \neq 1, R_{\nu}(\bar{\Lambda}) \grave{e}$ denso su $U_{s_{1}, s_{2}}^{r}(\mathcal{A})$, dove $v=r-2-\left[s_{1}\right]$.

E orvio che

$$
R_{p}(\bar{\Lambda}) \subset U_{s_{k}, s_{z}}^{r}(\Lambda)
$$

Assegnato inoltre $u \in U_{s_{1}, s_{2}}^{r}(\Lambda)$ poniamo:

$$
u^{\left(Q_{\nu}\right)}=u-\chi \sum_{|\gamma| \leqslant v} \frac{\partial^{\gamma} u(0,0)}{\gamma !} x^{\gamma} .
$$

Riesce in virtù del Lemma $3.1 u^{\left(Q_{y}\right)} \in W_{s_{1}, s_{2}}^{p}(\Lambda)$.

Esisterà allora, per il Teorema 8.1 di $[1]$ una successione $\left\{u_{n}\right\}$ di elementi di $D(\bar{A}-\{0\})$ tale che:

$$
\lim _{n \rightarrow \infty}\left\|u_{n}-u^{\left(Q_{\nu}\right)}\right\|_{W_{s_{1}, s_{2}}^{r}(A)}=0
$$

Quindi per il Lemma 2.1 si avrà anche:

$$
\lim _{n \rightarrow \infty}\left\|u_{n}-u^{\left(Q_{\nu}\right)}\right\|_{U_{s_{1}, s_{2}}^{r}(A)}=0
$$


Allora

$$
\left\{u_{n}+\chi \sum_{|y| \leqslant p} \frac{\partial^{\gamma} u(0,0)}{\gamma !} x^{\gamma}\right\}
$$

approssima $u(x)$ in $U_{s_{1}, s_{2}}^{r}(\Lambda)$.

Assegnato ancora un intero $v$ indichiamo adesso con $R_{y}([0,+\infty[)$ l'insieme di funzioni così definito:

$$
g(\varrho) \in R_{v}\left(\left[0,+\infty[) \Leftrightarrow \exists f \in \mathscr{D}\left(R_{+}\right)\right.\right.
$$

ed esiste un polinomio $Q_{y}(\varrho)$ di grado minore od uguale a $y$ tale che sia:

$$
g(\varrho)=f+\chi(\varrho) Q_{\nu}(\varrho)
$$

dove $\chi(\varrho)$ è un'assegnata funzione di classe $\mathfrak{D}([0,+\infty[)$ e tale che:

$$
\chi(\varrho) \begin{cases}=1 & \varrho \leqslant 1, \\ =0 & \varrho \geqslant 2 .\end{cases}
$$

E orvio che riesce:

$$
R_{v}([0,+\infty[) \subset \mathcal{D}([0,+\infty[)
$$

e che per $v^{\prime}<v^{\prime \prime}$ si ha:

$$
R_{v^{\prime}}\left(\left[0,+\infty[) \subset R_{v^{n}}([0,+\infty[)\right.\right.
$$

Vale il seguente teorema la cui dimostrazione è analoga a quella del Teorema 5.1.

TEOREMra 5.2. $-S e s_{1} \leqslant \frac{3}{2}, s_{1}+1 \leqslant r$ e $s_{1} \neq 1, R_{v}\left(\left[0,+\infty[)\right.\right.$ è denso su $U_{s_{1}+\frac{1}{2}, s_{2}+\frac{1}{2}}^{r-\frac{1}{2}}\left(R_{+}\right)$ dove $\nu=r-2$ per $s_{1}<1, v=r-3$ per $s_{1}>1$.

Detta $v_{k}$ la normale a $\sigma_{k}$ orientata verso l'interno di $A$ porremo:

$$
\gamma_{j k} u=\partial^{i} u / \partial \nu_{k / \sigma_{k}}^{i} \quad u \in R_{\nu}(\bar{\Lambda})
$$

Vale il seguente teorema di tracce:

TEOREMA 5.3. - Siano $s_{1} \in\left[0, \frac{3}{2}\left[; s_{1} \neq 1, s_{1}+1 \leqslant r, s_{2} \in R, s_{1} \leqslant s_{2}, r \geqslant 2\right.\right.$. L'applicazione che ad $u$ associa $\left\{\gamma_{{ }_{i n}} u\right\} k=1,2, j=0,1, \ldots, r-1$. definita $s u R_{p}(\bar{\Lambda})$ si prolunga per continuità in un'applicazione lineare e continua da $U_{s_{1}, s_{2}}^{r}(\Lambda)$ in

$$
\prod_{k=1,2} \prod_{j=0 \ldots \ldots ., r-1} U_{s_{1}, s_{2}}^{r-i-\frac{1}{2}}\left(\sigma_{k}\right)
$$


Il codominio di tale applicasione è la classe dei vettori $\left\{g_{j k}\right\} \in \prod_{k_{k}=1,2} \prod_{j=0 \ldots, r-1} U_{s_{1}, s_{2}}^{r-j-\frac{1}{2}}\left(\sigma_{k}\right)$ tali che esista un polinomio $Q_{y}(x)$ di grado $v=r-2-\left[s_{1}\right]$ per cui si abbia:

$$
\partial_{\varrho}^{i}\left(\left(\gamma_{j k} Q_{\nu}\right)_{k}\right)(0)=\left(\partial_{Q}^{i}\left(g_{j k}\right)_{k}\right)(0) \quad i \leqslant \nu-j
$$

Sia dato $u \in R_{y}(\bar{\Lambda})$ e sia:

$$
u^{\left(Q_{p}\right)}=u-\chi \sum_{|\alpha| \leqslant v} \frac{\partial^{\alpha} u(0,0)}{\alpha !} x^{\alpha}
$$

Si ha ovviamente:

$$
u^{\left(Q_{\vartheta}\right) \in W_{s_{1}, s_{2}}^{r}(\Lambda)}
$$

per il Lemma 3.1.

Dal Teorema 8.5 di [1], dal Teorema 2.1 e dal Lemma 3.1 segue:

$$
\begin{aligned}
& \left\|\gamma_{j k} u^{\left(Q_{\nu}\right)}\right\|_{W W_{s_{1}, s_{2}}^{r-j-\frac{1}{2}\left(\sigma_{k}\right)}} \leqslant c_{1}\left\|u^{\left(Q_{\nu}\right)}\right\|_{W_{s_{1}, s_{2}}^{r}(A)} \leqslant c_{2}\left\|u^{\left(Q_{\nu}\right)}\right\|_{U_{s_{1}, s_{2}}^{r}(\Lambda)} \leqslant \\
& \leqslant c_{3}\left(\|u\|_{U_{s_{1}, s_{8}}^{r}(A)}+\sum_{|\alpha| \leqslant y} \mid \partial^{\alpha} u(0,0) \|\right) \leqslant c_{4}\|u\|_{U_{s_{1}, s_{\mathrm{g}}(A)}} ;
\end{aligned}
$$

d'altro canto per il Lemma 4.1:

$$
\begin{aligned}
& \left\|\gamma_{j k} u\right\|_{U_{s_{1}, s_{k}}^{r-j-\frac{1}{2}}\left(\sigma_{k}\right)} \leqslant c\left(\left\|\gamma_{j k} u^{\left(Q_{p}\right)}\right\|_{U_{s_{1}, s_{2}}^{r-j-\frac{1}{2}\left(\sigma_{k}\right)}}+\sum_{|\alpha| \leqslant \mu} \mid \partial^{\alpha} u(0,0) \|\right) \leqslant \\
& \quad \leqslant c_{1}\left(\left\|\gamma_{j k} u^{\left(Q_{p}\right)}\right\|_{W_{s_{1}, s_{2}}^{r-j-\frac{1}{2}\left(\sigma_{k}\right)}}+\sum_{|\alpha| \leqslant \mu} \mid \partial^{\alpha} u(0,0) \|\right) \leqslant \epsilon_{2}\|u\|_{U_{s_{1}, s_{3}}^{r}(A)} .
\end{aligned}
$$

Dati inoltre $u \in U_{s_{1}, s_{3}}^{r}(\Lambda)$ e $\left\{g_{j k}\right\} \in \prod_{k=1,2} \prod_{j=0}^{r-1} U_{s_{1}, s_{2}}^{r-j-\frac{1}{2}}\left(\sigma_{k}\right)$ tali che $\gamma_{j k} u=g_{j k}$, risulta $u^{\left(Q_{p}\right)} \in$ $\in W_{s_{1}, s_{2}}^{r}(\Lambda)$ e quindi: $\gamma_{j k}\left(u^{\left(Q_{p}\right)}\right) \in W_{s_{1}, s_{z}}^{r-j=\frac{1}{2}}\left(\sigma_{k}\right)$. Da qui:

$$
\partial_{\varrho}^{\imath}\left(g_{j k}\right)(0)=\partial_{\varrho}^{i}\left(\left(\gamma_{j k} Q_{\nu}\right)_{k}\right)(0) \quad \text { per } i \leqslant \nu-j
$$

Sia adesso $\left\{g_{j k}\right\}$ un vettore appartenente a $\prod_{k=1,2} \prod_{j=0, \ldots, 1} U_{s_{1}, s_{2}}^{r-j-\frac{1}{2}}\left(\sigma_{k}\right)$ tale che esista un polinomio $Q_{\nu}(x)$ verificante le (5.1); poniamo:

$$
\varphi_{i k}=g_{i k}-\gamma_{i k}\left(\chi Q_{\eta}\right)
$$

riesce, in virtù del Lemma $4.2 \varphi_{j k} \in W_{s_{1}, \varepsilon_{2}}^{r-j-\frac{1}{2}}\left(\sigma_{k}\right)$; quindi per il Teorema 8.5 di [1] esiste una $u \in W_{s_{1}, s_{a}}^{r}(\Lambda)$ tale che:

$$
\begin{aligned}
& \gamma_{j k} u / \sigma_{k}=\varphi_{j k}, \\
& \|u\|_{j W_{s_{1}, s_{s}}^{r}(A)} \leqslant c \sum_{k=1}^{2} \sum_{j=0}^{r-1}\left\|\varphi_{j k}\right\|_{W_{s_{1}, \ell_{2}}^{r-j-\frac{1}{2}}\left(\sigma_{k}\right)} .
\end{aligned}
$$


Sfruttando il Teorema 4.1 ed il Lemma 4.2 si ha:

$$
\begin{aligned}
& \|u\|_{W_{s_{1}, s_{z}}^{r}(A)} \leqslant c_{1} \sum_{k=1}^{2} \sum_{j=0}^{r-1}\left\|\varphi_{j k}\right\|_{U_{s_{1}, s_{z}}^{r-j-\frac{\pi}{2}\left(\sigma_{k}\right)}} \leqslant \\
& \leqslant c_{2}\left(\sum_{k=1}^{2} \sum_{j=0}^{r-1}\left(\left\|g_{j k}\right\|_{U_{s_{1}, s_{2}}^{r-j-\frac{1}{2}}\left(\sigma_{k}\right)}+\sum_{i=0}^{p-j} \mid \partial^{i}\left(\left(g_{j k}\right)_{k}\right)(0) \|\right)\right) \leqslant c_{3} \sum_{k=1}^{2} \sum_{j=0}^{r-1}\left\|g_{j k}\right\|_{U_{s_{1}, g_{2}}^{r-j-\frac{1}{k}}\left(\sigma_{k}\right)} .
\end{aligned}
$$

Posto:

$$
v=u+\chi Q_{v}
$$

riesce:

e quindi per il Lemma 4.1:

$$
\gamma_{j k} v=g_{j k}
$$

$$
\begin{aligned}
& \|v\|_{U_{s_{1}, s_{2}}^{r}(A)} \leqslant 0\left(\|u\|_{U_{s_{1}, s_{2}}^{r}(A)}+\left\|\chi Q_{v}\right\|_{U_{s_{x}, s_{2}}^{r}(A)}\right) \leqslant \\
& \leqslant e^{\prime}\left(\|u\|_{W_{s_{1}, s_{2}}^{r}(A)}+\left\|\chi Q_{p}\right\|_{U_{s_{1}, s_{2}}^{r}(A)}\right) \leqslant c^{\prime \prime} \sum_{h=1}^{2} \sum_{j=0}^{r-1}\left\|g_{j k}\right\|_{U_{s_{1}, s_{2}}^{r-j-\frac{3}{3}\left(\sigma_{k}\right)}} .
\end{aligned}
$$

Si è tenuto conto più volte nel corso di tale dimostrazione che i coefficienti di $Q_{\nu}(x)$ possono esprimersi mediante le quantità $\left(\partial_{\varrho}^{i}\left(g_{j k}\right)_{k}\right)(0)$ in virtù delle (5.1).

Nota. - Esplicitiamo le condizioni (5.1) nel caso particolare in cui $\Lambda$ è un angolo retto e $\nu=r-2$.

Sia $\sigma_{1}$ l'asse delle $x ; \sigma_{2}$ l'asse delle $y$.

Posto:

$$
Q_{\nu}(x, y)=\sum_{n=0}^{r-2} \sum_{|\alpha|=n} a_{\alpha_{1}, \alpha_{2}} x^{\alpha_{1}} y^{\alpha_{2}}
$$

si trova con facili calcoli:

$$
\begin{aligned}
& \partial_{x}^{i}\left(\partial_{y}^{j} Q_{y}(x, y)\right) / \sigma_{1}(0)=j ! i ! a_{i, j}=\left(\partial_{x}^{i} g_{j, 1}\right)(0) \\
& \partial_{y}^{i}\left(\partial_{x}^{j} Q_{p}(x, y)\right) / \sigma_{2}(0)=j ! i ! a_{j, i}=\left(\partial_{y}^{i} g_{j, 2}\right)(0)
\end{aligned}
$$

Quindi in definitiva dovrà aversi :

$$
\left(\partial_{x}^{i} g_{j, 1}\right)(0)=\left(\partial_{u}^{j} g_{i, 2}\right)(0), \quad i+j \leqslant r-2 .
$$

Le $g_{r-1, k}$ soddisfano alle condizioni naturali derivanti dalla appartenenza a $W_{s_{1}, s_{2}}^{1-\frac{1}{2}}\left(\sigma_{k}\right)$ (cfr. anche [4]).

\section{6. - Alcuni lemmi di inclusione.}

LemMa 6.1. - Sia $s_{1}, s_{2} \in R$; riesce:

$$
\begin{aligned}
& u \in U_{s_{1}, s_{s}}^{r+|\alpha|}(\Lambda) \Rightarrow \partial^{\alpha} u \in U_{s_{1}, s_{2}}^{r}(\Lambda), \\
& \left\|\partial^{\alpha} u\right\|_{U_{s_{1}, s_{2}}^{r}(\Lambda)}^{r} \leqslant c\|u\|_{U_{s_{1}, s_{2}}^{r+\alpha\}}(\Lambda)} .
\end{aligned}
$$

8- Annali di Matematica 
Supponiamo $r \geqslant 2$; si ha:

$$
\begin{aligned}
& \left\|\partial^{\alpha} u\right\|_{U_{s_{1}, s \varepsilon}^{r}(\Lambda)}=\sum_{|\gamma| \leqslant r-2} \int_{A_{2}}\left|\partial^{\gamma+\alpha} u\right|^{2} d x+\sum_{|\beta|=r-1} \sum_{|\gamma| \leqslant 1} \int_{A_{2}}|x|^{2\left(s_{1}+|\gamma|-1\right)}\left|\partial^{\gamma+\alpha+\beta} u\right|^{2} d x+
\end{aligned}
$$

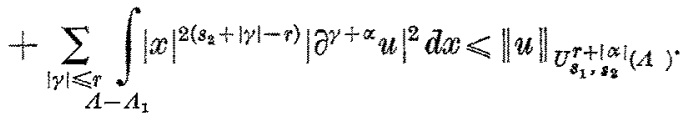

LEMMA 6.2. - Se $s_{1}, s_{2}, t_{1}, t_{2} \in R$ ed è:

$$
s_{1} \leqslant t_{1}, \quad s_{\mathrm{z}} \geqslant t_{2}
$$

riesee:

$$
\|u\|_{U_{t_{1}, t_{z}}^{r}(A)} \leqslant c\|u\|_{U_{s_{1}, s_{2}}^{r}(A)}
$$

ciò̀:

$$
U_{s_{1}, s_{2}}^{r}(\Lambda) \subset U_{t_{1}, t_{2}}^{r}(\Lambda)
$$

La dimostrazione di ciò è immediata.

Lemara 6.3. - Assegnati $k, r \in N, s_{1} \in R_{+}$per ogni $\alpha \leqslant 1$ se $k=1$ e per ogni $\alpha \in R$ se $k \geqslant 2$, per ogni $t \geqslant 0$ riesce:

$$
U_{B_{1}+\alpha, s_{2}+k+t}^{r+k}(\Lambda) \subset U_{s_{1}, s_{2}}^{s}(\Lambda)
$$

algebricamente e topologicamente.

Se $k=1$ basta osservare che per il Lemma 1.4:

$$
\begin{aligned}
\int_{\Lambda_{1}}|x|^{2\left(s_{1}-1\right)}\left|\partial^{r-1} u\right|^{2} d x \leqslant c\left(\int_{\Lambda_{2}}|x|^{2 s_{1}}\left|\partial^{r} u\right|^{2} d x+\right. & \\
& \left.+\int_{\Lambda_{2}}\left|\partial^{r-1} u\right|^{2} d x\right) \leqslant c^{\prime}\left(\int_{\Lambda_{2}}|x|^{2\left(s_{1}+\alpha-1\right)}\left|\partial^{r} u\right|^{2} d x+\int_{\Lambda_{2}}\left|\partial^{r-1} u\right|^{2} d x\right) .
\end{aligned}
$$

Se $k \geqslant 2$ basta osservare che per il Lemma 1.4 si ha:

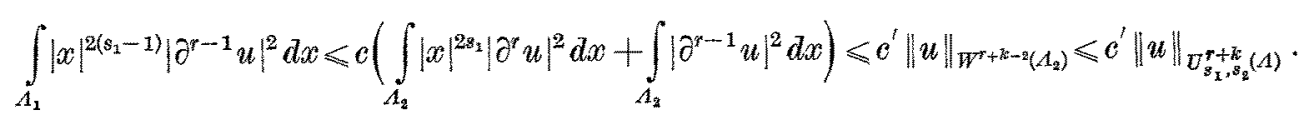

Lemma 6.4. - Assegnati $r, k \in N, s_{1}, s_{2} \in R$ si ha:

$$
g \in U_{s_{1}+\frac{1}{2}, s_{2}+\frac{1}{2}}^{r+k}\left(R_{+}\right) \Rightarrow \partial^{k} g \in U_{s_{1}+\frac{1}{2}, s_{2}+\frac{1}{2}}^{r-\frac{1}{2}}\left(R_{+}\right)
$$

ed inoltre:

$$
\left\|\partial^{k} g\right\|_{U_{s_{1}+\frac{1}{2}, s_{2}+\frac{1}{2}}^{r-\frac{1}{2}\left(R_{+}\right)}} \leqslant c\|g\|_{U_{s_{1}+\frac{1}{2}, s_{2}+\frac{1}{2}}^{r+\frac{1}{2}\left(R_{+}\right)}} .
$$


Basta osservare che:

$$
\sum_{i=0}^{r-2} \int_{0}^{2}\left|g^{(i+k)}\right|^{2} d \varrho \leqslant \sum_{i=0}^{r+k-2} \int_{0}^{2}\left|g^{(i)}\right|^{2} d \varrho
$$

e che:

$$
\sum_{i=0}^{r-1} \int_{1}^{+\infty} \varrho^{2\left(s_{2}+i+k-(r+k-1)-\frac{1}{k}\right)}\left|g^{(i+k)}\right|^{2} d \varrho \leqslant \sum_{j=0}^{r+k-1} \int_{i}^{+\infty} Q^{2\left(s_{2}+j-(r+k-1)-\frac{1}{2}\right)}\left|\partial^{j} g\right|^{2} d \varrho .
$$

LEMMA 6.5. - Assegnati $s_{1}, s_{2}, t_{1}, t_{2} \in R$ se riesce $s_{1} \leqslant t_{1}$ ed $s_{2} \geqslant t_{2}$ si $h a$ :

$$
U_{s_{1}+\frac{1}{2}, s_{2}+\frac{1}{2}}^{r-\frac{1}{2}}\left(R_{+}\right) \subset U_{t_{3}+\frac{2}{2}, t_{2}+\frac{1}{2}}^{r-\frac{1}{2}}\left(R_{+}\right)
$$

algebricamente e topologicamente.

Procedendo come nella dimostrazione della $(9.4)$ di [1] si ottiene:

$$
\begin{aligned}
& \int_{-\infty}^{\log 2} \int_{-\infty}^{\log 2} \frac{\left|e^{t_{1} y} g^{(r-1)}\left(e^{y}\right)-e^{l_{1} y^{\prime}} g^{(r-1)}\left(e^{y^{\prime}}\right)\right|^{2}}{\left|y-y^{\prime}\right|^{2}} d y d y^{\prime} \\
& \leqslant c\left(\int_{-\infty}^{\log 2} \int_{-\infty}^{\log 2} \frac{\left|e^{s_{1} y} g^{(r-1)}\left(e^{y}\right)-e^{s_{1} y^{\prime}} g^{(r-1)}\left(e^{y^{y}}\right)\right|^{2}}{\left|y-y^{\prime}\right|^{2}} d y d y^{2}+\int_{-\infty}^{\log 2}\left|e^{s_{1} y} g\left(e^{y}\right)\right|^{2} d y\right) .
\end{aligned}
$$

Usando la (9.3) di [1] unitamente a quest'ultima diseguaglianza si ricava la tesi.

Lemma 6.5. - Assegnati $r \in N, s_{1} \in R_{+}, s_{2} \geqslant s_{1}$ riesce:

$$
U_{s_{1}+1+\frac{1}{2}, s_{2}+1+\frac{1}{2}}^{r+1-\frac{1}{2}}\left(R_{+}\right) \subset U_{s_{1}+\frac{1}{2}, s_{2}+\frac{1}{2}}^{r-\frac{1}{2}}\left(R_{+}\right)
$$

algebricamente e topologicamente.

Per semplicità ci limiteremo a considerare il caso $\left.s_{1} \in\right] 0, \frac{1}{2}[, r \geqslant 2$.

Sia $g \in U_{s_{1}+1+\frac{1}{2} s_{2}+1+\frac{1}{3}}^{r+1-R_{+}}\left(R_{+}\right)$.

Poniamo:

$$
g^{\left(Q_{p}\right)}=g-\chi \sum_{\gamma \leqslant r-2} \frac{\partial^{\gamma} g(0)}{\gamma !} \varrho^{\gamma}
$$

con

$$
\chi(\varrho)\left\{\begin{array}{ll}
=1 & \text { se } \varrho \leqslant 1, \\
=0 & \text { se } \varrho \geq 2,
\end{array} \quad \text { e } \chi \in \mathfrak{D}([0,+\infty[),\right.
$$


(osserva che $g$ è dotata di tracce con le sue derivate fino all'ordine $r-2$ poichè è $\left.1<s_{1}+1<\frac{3}{2}\right)$.

In virtù del Lemma 4.2 riesce:

$$
g^{\left(Q_{p}\right)} \in W_{s_{1}+1+\frac{1}{2}, s_{2}+1+\frac{1}{2}}^{r+1-\frac{1}{2}}\left(R_{+}\right) \subset W_{s_{2}+\frac{1}{2}, s_{2}+\frac{1}{2}}^{r-\frac{1}{2}}\left(R_{+}\right) \simeq U_{s_{1}+\frac{1}{2}, s_{2}+\frac{1}{2}}^{r-\frac{1}{2}}\left(R_{+}\right)
$$

Quindi per il Lemma 4.2 ed il Teorema 4.1:

$$
\begin{aligned}
& \|g\|_{U_{s_{1}+\frac{1}{2}, s_{2}+\frac{1}{2}}^{r-\frac{1}{2}}\left(R_{+}\right)} \leqslant\left\|g^{\left(Q_{\eta}\right)}\right\|_{U_{s_{1}+\frac{1}{2}, s_{2}+\frac{1}{2}}^{r-\frac{1}{2}}\left(R_{+}\right)}+ \\
& +c \sum_{\gamma \leqslant r-2}\left|\partial^{\gamma} g(0)\right| \leqslant c_{1}\left(\left\|g^{\left(Q_{p}\right)}\right\|_{U_{s_{1}+\frac{1}{2}, s_{2}+\frac{1}{2}}^{r+1}\left(R_{+}\right)}+\sum_{\gamma \leqslant r-2} \mid \partial^{\gamma} g(0)\left\|\leqslant c_{2}\right\| g \|_{U_{s_{1}+1+\frac{1}{2}+\frac{1}{2}, s_{2}+1+\frac{1}{2}}^{r+1}\left(R_{+}\right)} .\right.
\end{aligned}
$$

\section{7. - Alcuni lemmi di immersione compatta.}

Lemma 7.1. - Siano dati $r \in N_{0}, s_{1} \in R_{+}, s_{2} \in R, \varepsilon \in R_{+}$, con le ulteriori condizioni: $s_{1} \leqslant s_{2}, s_{1}<\varepsilon+1, s_{1} \leqslant 2, s_{1} \neq \varepsilon$ se $r \geqslant 1$; allora l'immersione di $U_{s_{1}+1-\varepsilon_{,}+s_{2}+1+\varepsilon}^{r+1}(\Lambda)$ in $U_{s_{1}, s_{1}}^{r}(\Lambda)$ è compatta.

Se $r=01$ asserto segue dal Teorema 8.8 di [1].

Se $s_{1}>\varepsilon$ si ha:

$$
U_{s_{1}+1-\varepsilon, s_{2}+1+\varepsilon}^{2}(\Lambda) \simeq W_{s_{1}+1-\varepsilon, s_{2}+1+\varepsilon}^{2}(\Lambda)
$$

per cui nel caso $r=1$ e $s_{1}>\varepsilon$ la tesi segue ancora dal Teor. 8.8 di [1].

Che sia $U_{s_{1}+1-8, s_{2}+1+\varepsilon}^{++1}(\Lambda) \subset U_{s_{1}, s_{2}}^{r}(\Lambda)$ segue dai Lemmi 6.2 e 6.3 .

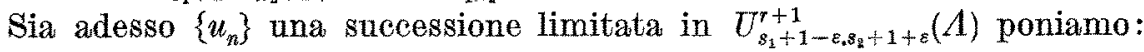

$$
u_{n}^{\left(Q_{\eta}\right)}=u_{n}-\chi(x) \sum_{|\gamma| \leqslant \nu} \frac{\partial^{p} u_{n}(0,0)}{\gamma !} x^{\gamma}
$$

con $\chi(x) \in \mathfrak{D}(\bar{\Lambda})$ e tale che sia:

$$
\chi(x) \begin{cases}=1 & \text { se }|x| \leqslant 1, \\ =0 & \text { se }|x| \geqslant 2,\end{cases}
$$

e con $\nu=r-1-\left[s_{1}+1-\varepsilon\right]$.

Per il Lemma 3.1 riuscirà $\left\{u_{n}^{Q_{n}}\right\}$ limitata in $W_{s_{1}+1-\varepsilon, s_{2}+1+\varepsilon}^{s+1}(\Lambda)$; quindi per il Teor. 8.8 di [1] da tale successione se ne potrà estrarre un'altra convergente in $W_{3_{2} s_{8}}^{r}(\Lambda)$ che denoteremo ancora con lo stesso simbolo.

Esisterà, quindi, $u \in W_{s_{2}, s_{2}}^{r}(\Lambda)$ tale che riesca:

$$
\lim _{n \rightarrow \infty}\left\|u_{n}^{\left(Q_{y}\right)}-u\right\|_{w_{s_{1}, s_{2}}^{*}(A)}=0
$$


Osserviamo che, in virtù del Teor. 2.1, riesce:

$$
\left|\frac{\hat{o}^{\gamma} u_{n}(0,0)}{\gamma !}\right| \leqslant c\left\|u_{n}\right\|_{U_{s_{1}+1-\varepsilon, s_{2}+1+\varepsilon}^{r+1}+(1)} \leqslant c \cdot k
$$

quindi dalla successione $\left\{\left(\partial^{\gamma} u_{n}(0,0)\right) / \gamma !\right\}$ se ne potrà estrarre un'altra convergente al numero $O_{\gamma}$.

Allora, tenendo presente il tuemma 2.1, si avrà:

$$
\begin{aligned}
& \left\|u_{n}-\chi \sum_{i y \mid \leqslant \nu} C_{y} x^{y}-u\right\|_{\delta_{s_{1}, s_{2}}^{r}(A)} \\
& =\left\|u_{n}-\chi \sum_{|\gamma| \leqslant p} C_{\gamma} x^{\gamma}-u+\chi \sum_{|\gamma| \leqslant p} \frac{\partial v_{u_{n}}(0,0)}{\gamma !} x^{\gamma}-\chi \sum_{|\gamma| \leqslant p} \frac{\partial^{\gamma} u_{n}(0,0)}{\gamma !} x^{\gamma}\right\|_{v_{s_{1}, s_{\mathrm{z}}}^{*}(A)} \\
& \leqslant\left\|u_{n}^{\left(Q_{\eta}\right)}-u\right\|_{w_{s_{1}, z_{3}}^{r}(A)}+c \sum_{|\gamma| \leqslant p}\left|\frac{\partial y u_{n}(0,0)}{\gamma !}-C_{\gamma}\right|
\end{aligned}
$$

e da qui l'asserto.

LEMMa 7.2. - Siano assegnati $r \in N, s_{1}, \varepsilon \in R_{+}, s_{2} \in R$ in modo tale che sia: $s_{1} \leqslant s_{2}$, $s_{1}<\frac{3}{2}, s_{1}+1-\varepsilon \leqslant \frac{3}{2}, s_{1} \neq \varepsilon ;$ si ha allora che l'immersione $d i \quad U_{s_{1}+1-\varepsilon+\frac{1}{2}, s_{2}+1+\varepsilon+\frac{1}{2}}^{r+1-\frac{1}{2}}\left(R_{+}\right)$ in $U_{s_{1}+\frac{1}{2}, s_{2}+\frac{1}{2}}^{r-\frac{\hbar}{2}}\left(R_{+}\right)$è compatta.

Dimostrazione analoga alla precedente.

DEFINIZIONe 7.1. $-\forall(\alpha, \beta) \in R^{2}$ poniamo:

$$
\|u\|_{\mathfrak{L}_{\alpha, \beta}^{2}}^{2}=\int_{\Lambda_{1}}|x|^{2 \alpha}|u|^{2} d x+\int_{A-\Lambda_{1}}|x|^{2 \dot{\beta}}|u|^{2} d x .
$$

Lema 7.3. - Assegnati $s_{1}, s_{2} \in R, s_{1} \leqslant s_{2}, r \in N, r \geqslant 2$ con $s_{1} \leqslant 1$ se $r=2, \forall \varepsilon>0$ l'immersione di $U_{s_{1}, s_{2}}^{r}(A)$ in $\mathcal{L}_{0, s_{3}-r-\varepsilon}^{2}$ è compatta.

Sia $\left\{u_{n}\right\}$ una successione di elementi di $U_{s_{1}, s_{3}}^{r}(A)$ tale che riesca:

$$
\left\|u_{n}\right\|_{\delta_{s_{t}, s_{2}}^{r}(A)} \leqslant M \quad \forall n \in N
$$

Si avrà in particolare

$$
\begin{aligned}
& \left\|u_{n}\right\|_{W^{2}\left(A_{2}\right)} \leqslant M, \\
& \|u\|_{W_{s_{2}}^{r}\left(A-A_{1}\right)} \leqslant M .
\end{aligned}
$$


Poichè l'immersione di $W^{1}\left(\Lambda_{2}\right)$ in $L^{2}\left(\Lambda_{2}\right)$ è compatta, per la (7.2) da $\left\{u_{n}\right\}$ si potrà estrarre una successione $\left\{u_{n}^{\prime}\right\}$ convergente ad $u^{\prime}$ in $L^{2}\left(\Lambda_{2}\right)$; ciò̀

$$
\lim _{n \rightarrow \infty}\left\|u_{n}^{\prime}-u^{\prime}\right\|_{L^{2}\left(A_{2}\right)}=0 \text {. }
$$

Siano $\zeta_{1}(x)$ e $\zeta_{2}(x)$ due funzioni di classe $\mathscr{D}(\bar{\Lambda})$ tali che sia:

$$
\begin{array}{ll}
\zeta_{1}(x)=0 & \text { per }|x| \geqslant 2, \\
\zeta_{2}(x)=0 & \text { per }|x| \leqslant 1, \\
\zeta_{1}(x)+\zeta_{2}(x)=1 & \forall x \in \bar{\Lambda} .
\end{array}
$$

Ovviamente in $L^{2}\left(\Lambda_{2}\right)\left\{\zeta_{1} u_{n}^{\prime}\right\}$ converge a $\zeta_{1} u^{\prime}$.

D'altro canto si ha:

$$
\left\|\zeta_{1} u_{n}^{\prime}-\zeta_{1} u^{\prime}\right\|_{\mathfrak{E}_{0 . s_{2}-r-\xi}^{2}} \leqslant c\left\|\zeta_{1} u_{n}^{\prime}-\zeta_{1} u^{\prime}\right\|_{L^{2}\left(\Lambda_{2}\right)}
$$

e quindi $\left\{\zeta_{1} u_{n}^{\prime}\right\}$ converge a $\zeta_{1} u^{\prime}$ anche in $\mathfrak{L}_{0, s_{3}-r-\varepsilon}^{2}$.

D'altro canto per la (7.3):

$$
\left\|\zeta_{2} u_{n}^{\prime}\right\|_{W_{s_{2}}^{*}(A)} \leqslant c \cdot M
$$

Inoltre per il Teorema $8.7 \mathrm{di}[1] W_{s_{2}}^{r}(A)$ si immerge in modo compatto in

$$
L_{s_{2}-r, \varepsilon}^{2}(\Lambda) \simeq L_{s_{2}-r+\varepsilon}^{2}\left(A_{1}\right) \cap L_{s_{2}-r-\varepsilon}^{2}\left(\Lambda-A_{1}\right)
$$

(efr. 6.4 di [1] e per la definizione di $L_{s, \lambda}^{P}$ efr. [1] paragrafo 6 ); quindi poichè:

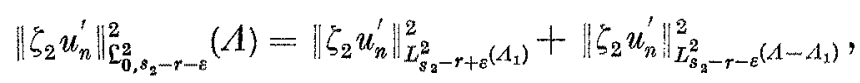

dalla successione $\left\{u_{n}^{\prime}\right\}$ se ne potrà estrarre un'altra $\left\{u_{n}^{\prime \prime}\right\}$ tale che $\zeta_{2} u_{n}^{\prime \prime}$ converga a $\zeta_{2} u^{\prime \prime}$ in $\mathrm{c}_{0 . s_{2}-r-\varepsilon}^{2}$.

In definitiva:

$$
v_{n}=\zeta_{1} u_{n}^{\prime \prime}+\zeta_{2} u_{n}^{\prime \prime} \quad \text { converge a } \zeta_{1} u^{\prime}+\zeta_{2} u^{\prime \prime} \text { in } \mathfrak{L}_{0, s_{2}-r \cdots \varepsilon}^{2} .
$$

\section{8. - Ipotesi e risultati per il problema.}

Sia dato un operatore differenziale lineare su $\Lambda$ del tipo:

$$
A=A(x, \partial)=\sum_{|\mu| \leqslant 2 m} a_{\mu}(x) \partial^{\mu}
$$


e per $k=1,2$ sia dato su $\sigma_{k}$ un sistema di $m$ operatori differenziali lineari di frontiera del tipo:

$$
B_{j k}=B_{j k}(x, \partial)=\sum_{|\mu| \leqslant m_{j k}} b_{j k \mu}(x) \partial^{\mu}, \quad j=1, \ldots, m .
$$

Supporremo che

$$
b_{j k \mu}=\stackrel{\circ}{b}_{j k \mu} / \sigma_{k}
$$

intendendo con ciò che i $b_{j k \mu}$ siano tracce su $\sigma_{k}$ (nel senso del Teorema 5.3) di funzioni $\stackrel{\circ}{b k \mu}_{j k}$ appartenenti ad opportuni spazi funzionali.

Facciamo le seguenti ipotesi:

$\left.H_{1}\right)$ i coefficienti $a_{\mu}(x)$ con $|\mu|=2 m e \stackrel{\circ}{b k \mu}(x)^{\prime}|\mu|=m_{j k}$ siano di classe $C^{0}(\bar{\Lambda})$ e convergenti all'infinito;

$\left.H_{2}\right) A$ è propriamente ellittico in $\bar{A}$ ed all'oo e, per ogni $k \in\{1,2\}$ gli operatori $B_{1 k}, \ldots, B_{m k}$ verificano la condizione complementare rispetto ad $A$ su $\sigma_{k}$ ed all' $\infty$; indicato con $A^{\prime}=A^{\prime}(x, \partial)$ e $B_{j k}^{\prime}=B_{j k}^{\prime}(x, \partial)$ le parti dominanti degli operatori $A$ e $B_{i k}$, sia $\Sigma_{1}$ il sottoinsieme discreto e numerabile di $R$ definito dal Teorema 1.1 di [2] relativamente agli operatori a coefficienti costanti:

$$
A^{\prime}(0, \partial), \quad B_{j k}^{\prime}(0, \partial), \quad k=1,2 ; j=1,2, \ldots, m,
$$

sia ancora $\Sigma_{2}$ il sottoinseme discreto e numerabile di $R$ definito dal Teorema 1.1 di [2] relativamente agli operatori a coefficienti costanti:

$$
\sum_{|\mu|=2 m}\left(\lim _{x \rightarrow \infty} a_{\mu}(x)\right) \partial^{\mu}, \quad \sum_{|\mu|=m m_{i k}}\left(\lim _{x \rightarrow \infty} b_{j k \mu}(x)\right) \partial^{\mu} \quad k=1,2 ; j=1,2, \ldots, m .
$$

Assegniamo $r \in N, r \geq \sup \left\{2 \mathrm{~m}, m_{j k}+\frac{1}{2}\right\}, s_{1} \in\left(\left[0, \frac{3}{2}[-\{1\}), s_{2} \in R, s_{1} \leqslant s_{2}\right.\right.$ e supponiamo ancora che:

$$
\begin{aligned}
& \left.H_{3}\right) \quad \partial^{\alpha} a_{\mu} \in L^{\infty}\left(\Lambda_{2}\right) \text { per } \begin{cases}|\mu|=2 m, & |\alpha| \leqslant r-2 m, \\
|\mu| \leqslant 2 m-1, & |\alpha| \leqslant r-2 m+1,\end{cases} \\
& \partial^{\alpha} \stackrel{\circ}{b j k}_{j k} \in L^{\infty}\left(\Lambda_{2}\right) \quad \text { per } \begin{cases}|\mu|=m_{j k}, & |\alpha| \leqslant r-m_{j k}, \\
|\mu| \leqslant m_{j k}-1, & |\alpha| \leqslant r-m_{j k}+1,\end{cases}
\end{aligned}
$$

se $s_{1}=0$ :

$$
\begin{array}{ll}
\lim _{x \rightarrow 0} \partial^{\alpha} a_{\mu}=0 & \operatorname{per} \begin{cases}|\mu|=2 m, & 0<|\alpha| \leqslant r-2 m-1 \\
|\mu| \leqslant 2 m-1, & 0 \leqslant|\alpha| \leqslant r-2 m-1\end{cases} \\
\lim _{x \rightarrow 0} \partial^{\alpha} \stackrel{\circ}{b k \mu}_{j k}=0 & \operatorname{per} \begin{cases}|\mu|=m_{j k}, & 0<|\alpha| \leqslant r-m_{j k}-1 \\
|\mu| \leqslant m_{j k}-1, & 0 \leqslant|\alpha| \leqslant r-m_{j k}-1,\end{cases}
\end{array}
$$




$$
\begin{aligned}
& \left.H_{3}^{\varepsilon}\right) \quad|x|^{2 m-|\mu|+|\alpha|} \partial^{\alpha} a_{\mu} \in L^{\infty}\left(\Lambda-A_{1}\right) \quad \text { per } \begin{cases}|\mu|=2 m, & |\alpha| \leqslant r-2 m, \\
|\mu| \leqslant 2 m-1, & |\alpha| \leqslant r-2 m+1\end{cases} \\
& |x|^{m_{j k}-|\mu|+|\alpha|} \partial^{\alpha} b_{j k \mu} \in L^{\infty}\left(\Lambda-\Lambda_{1}\right) \quad \text { per } \begin{cases}|\mu|=m_{j k}, & |\alpha| \leqslant r-m_{j k}, \\
|\mu| \leqslant m_{j k}-1, & |\alpha| \leqslant r-m_{j k}+1\end{cases}
\end{aligned}
$$

esiste inoltre un $\varepsilon \in[0,1]$ tale che sia:

$$
\begin{aligned}
& |x|^{2 m-|\mu|+\varepsilon} a_{\mu} \in L^{\infty}\left(\Lambda-\Lambda_{1}\right) \quad \text { per }|\mu| \leqslant 2 m-1, \\
& |x|^{\left|m_{j k}-\right| \mu \mid+\varepsilon} \stackrel{\circ}{b j k \mu}_{j k} \in L^{\infty}\left(\Lambda-\Lambda_{1}\right) \text { per }|\mu| \leqslant m_{j k}-1 ;
\end{aligned}
$$

$\left.H_{4}\right)$

$$
\begin{gathered}
\lim _{|\alpha| \rightarrow \infty}|| x|| \alpha\left|\partial^{\alpha} a_{\mu}(x)\right|=\lim _{|x| \rightarrow \infty}|| x|| \alpha\left|\partial^{\alpha} \stackrel{\circ}{b k \mu}_{j k}(x)\right|=0 \\
\operatorname{per} \begin{cases}|\mu|=2 m, & 0<|\alpha| \leqslant r-2 m, \\
|\mu|=m_{j k}, & 0<|\alpha|<r-m_{j k} .\end{cases}
\end{gathered}
$$

Poniamo:

$$
E_{r, s_{1}, s_{2}}(\Lambda)=W_{s_{1}, s_{2}}^{r-2 m}(\Lambda) \times \prod_{k=1}^{2} \prod_{j=1}^{m} W_{s_{1}, s_{2}}^{r-m_{j k}-\frac{1}{2}}\left(\sigma_{k}\right)
$$

In forza delle ipotesi $H_{3}$ ) e $H_{3}^{0}$ ) si verifica facilmente che l'operatore:

$$
\mathcal{A}: u \rightarrow\left(A u, B_{11} u / \sigma_{1}, \ldots, B_{m 2} u / \sigma_{2}\right)
$$

che va da $W_{s_{1}, s_{2}}^{r}(\Lambda)$ in $E_{r, s_{1}, s_{2}}(\Lambda)$ è lineare e continuo (cfr. anche [3]).

Poniamo ancora:

$$
G_{r, s_{1}, \varepsilon_{3}}(A)=U_{s_{1}, s_{3}}^{r-2 m}(A) \times \prod_{j=1}^{m} \prod_{k=1}^{2} U_{s_{1}, s_{3}}^{r-m_{3}-\frac{1}{2}}\left(\sigma_{k}\right)
$$

si verifica che:

LEMMA 8.1. - Nelle ipotesi $H_{3}$ ) e $H_{3}^{0}$ ) l'operatore $\mathcal{A}$ che va da $U_{s_{1}, s_{2}}^{r}(\Lambda)$ in $G_{r, z_{1}, s_{2}}(\Lambda)$ ò lineare e continuo.

Se $s_{1}>0$ la verifiea è immediata. Sia $s_{1}=0$.

Consideriamo, con $|\beta|=r-2 m-1$

$$
\int_{\Lambda_{1}}|x|^{-2}\left|\partial^{\beta}\left(a_{\mu} \partial^{\mu} u\right)\right|^{2} d x=\sum_{\alpha \leqslant \beta}(\beta) \int_{\lambda_{1}} \alpha|x|^{-2}\left|\partial^{\beta-\alpha} a_{\mu} \cdot \partial^{\mu+\alpha} u\right|^{2} d x .
$$


Se $|\mu|+|\alpha|=r-1$ questo termine si maggiora con

$$
K \int_{A_{1}}|x|^{-2}\left|\partial^{r-1} u\right|^{2} d x
$$

(Perchè ciò accada deve essere $|\beta|-|\alpha|=0$ e $|\mu|=2 m$ ).

Sia $|\mu|+|\alpha|<r-1$. Per il Teorema $2.1 \partial^{\mu+\alpha} u$ ha la traccia nell'origine.

Posto

$$
v(x)=\partial^{\beta-\alpha} a_{\mu} \cdot \partial^{\mu+\alpha} u
$$

si ha in forza dell'ipotesi $\left.H_{3}\right)$ :

$$
v(0)=0
$$

Dal Lemma 1.5 si ha:

$$
\begin{aligned}
\int_{A_{1}}|x|^{-2}\left|\partial^{\beta-\alpha} a_{\mu} \cdot \partial^{\mu+\alpha} u\right|^{2} d x \leqslant c \int_{A_{3}}|x|^{-2-\varepsilon}\left|\partial^{\beta-\alpha} a_{\mu} \cdot \partial^{\mu+\alpha} u\right|^{2} d x \leqslant \\
\quad \leqslant c^{\prime}\left(\int_{A}|x|^{-\varepsilon}\left|\partial^{k} a_{\mu}\right|^{2} \cdot\left|\partial^{\mu+\alpha} u\right|^{2} d x+\int_{A}|x|^{-\varepsilon}\left|\partial^{\beta-\alpha} a_{\mu}\right|^{2} \cdot\left|\partial^{k^{\prime}} u\right|^{2} d x\right.
\end{aligned}
$$

$\operatorname{con} k=|\beta-\alpha|+1$ e $k^{\prime}=|\mu+\alpha|+1$.

Adoperando l'ipotesi $H_{3}^{\varepsilon}$ ) per maggiorare questi ultimi integrali in $A-A_{1}$ ed applicando il Lemma 1.4 a

$$
\int_{\Lambda_{1}}|x|^{-\varepsilon}\left|\partial^{k+\alpha} u\right|^{2} d x
$$

e se necessario a

$$
\int_{\Lambda_{1}}|x|^{-\varepsilon}\left|\partial^{k^{\prime}} u\right|^{2} d x
$$

si ha l'asserto.

Vale il segunte teorema:

Teorema 8.1. - Nelle ipotesi poste, ma con $s_{1} \in\left[0,1\left[, s_{1}-r+1 \notin \Sigma_{1}, s_{2}-r+1 \notin \Sigma_{2}\right.\right.$ vale la seguente formula di maggiorazione, con $\varepsilon$ definito dall'ipotesi $B_{3}^{\varepsilon}$ ):

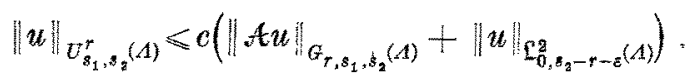

La dimostrazione di questo teorema sarà data al numero seguente.

Osserviamo intanto che dal Teorema 8.1, dal Lemma 7.3, e dal Lemma 5.1 pag. 171 di [5] si ha il seguente:

Corollario 8.1. - Nelle ipotesi del Teorema 8.1 con $\varepsilon>0$ l'immagine dell'operatore $A$ in $G_{r, s_{1}, s_{\mathrm{s}}}(\Lambda)$ è chiusa ed it suo nucleo $\grave{e}$ di dimensione finita. 


\section{9. - Dimostrazione del teorema 8.1.}

Lemina 9.1. - Fissati $r \in N, r \geqslant 2, s_{1} \in\left[0,1\left[s_{2} \leqslant s_{1}\right.\right.$, si ha per $|\alpha| \leqslant r-2$ e $\left.\lambda \in\right] 0,1[$ :

$$
\left|\partial^{\alpha} u(0,0)\right| \leqslant \lambda\|u\|_{U_{s_{1}, s_{\mathrm{a}}(\lambda)}^{r}}+c(\lambda)\|u\|_{L^{2}\left(A_{1}\right)} .
$$

I) Sia $|\alpha| \leqslant r-4$.

La (1.6) scritta per $t=(0,0)$ unitamente all'ipotesi $s_{1} \geqslant 0$ ed alle note formule di Erling da:

$$
\begin{aligned}
& \left|\partial^{\alpha} u(0,0)\right|^{2} \leqslant c \sum_{|\beta| \leqslant r-2} \int_{\Lambda_{1}}|x|^{2 s_{1}}\left|\partial^{\beta} u\right|^{2} d x \leqslant c_{1}\|u\|_{W^{r-2}\left(\Lambda_{1}\right)} \leqslant c_{1}\left(\lambda \int_{A_{1}}\left|\partial^{r-1} u\right|^{2} d x+c(\lambda)\|u\|_{L^{2}\left(\Lambda_{1}\right)}^{2}\right) \leqslant \\
& \leqslant c_{2} \cdot \lambda \int_{\Lambda_{2}}|x|^{2\left(\beta_{2}-1\right)}\left|\partial^{r-1} u\right|^{2} d x+e^{\prime}(\lambda)\|u\|_{L^{2}\left(\Lambda_{1}\right)}^{2} \leqslant c_{2} \cdot \lambda\|u\|_{U_{s_{1}, s_{2}(A)}^{\tau}}^{2}+e^{\prime}(\lambda)\|u\|_{L^{2}\left(\Lambda_{1}\right)}^{2} .
\end{aligned}
$$

II) $\operatorname{Sia}|\alpha|=r-3$.

Scritta la (1.6) per $t=(0,0), \alpha+\frac{1}{2}=s_{1}$ e $v(x)=u(\varepsilon x)$ si ha:

$$
\left|\partial^{r-3} v(0,0)\right|^{2} \leqslant c\left(\int_{\Lambda_{1}}|x|^{2 s_{1}}\left|\partial^{r-3} v\right|^{2} d x+\int_{A_{1}}|x|^{2 s_{1}}\left|\partial^{r-2} v\right|^{2} d x+\int_{\Lambda_{1}}|x|^{2 s_{1}}\left|\partial^{r-1} v\right|^{2} d x\right) .
$$

Osservando che:

$$
\partial_{x_{i}}^{k} v(x)=\partial_{t_{i}}^{k} u \cdot \varepsilon^{k}
$$

si ha, effettuando il cambiamento di variabili $\varepsilon x=t$ e ricordando che $s_{1}-1<0 \leqslant s_{1}$ :

$$
\begin{aligned}
\left|\partial^{r-3} u(0,0)\right|^{2} \leqslant \varepsilon^{-2\left(s_{1}+1\right)} & \int_{\Lambda_{1}}\left|\partial^{r-3} u\right|^{2} d t+\varepsilon^{-2 \varepsilon_{1}} \int_{\Lambda_{1}}\left|\partial^{r-2} u\right|^{2} d t+\varepsilon^{-2\left(s_{2}-1\right)} \int_{\Lambda_{1}}|t|^{2\left(s_{1}-1\right)}\left|\partial^{r-1} u\right|^{2} d t \leqslant \\
& \leqslant \varepsilon^{-2\left(s_{1}+1\right)}\left(\int_{\Lambda_{1}}\left(\left|\partial^{r-3} u\right|^{2}+\left|\partial^{r-2} u\right|^{2}\right) d t\right)+\varepsilon^{-2\left(s_{1}-1\right)} \int_{\Lambda_{1}}|t|^{2\left(s_{1}-1\right)}\left|\partial^{r-1} u\right|^{2} d t
\end{aligned}
$$

dove si è scelto $0<\varepsilon<k<1$ con $k$ da fissarsi opportunamente.

Applicando ai primi due integrali la diseguaglianza di Erling con $\lambda=\varepsilon^{2\left(s_{1}+1\right)+1}$ si ha:

$$
\begin{array}{r}
\left|\partial^{r-3} u(0,0)\right|^{2} \leqslant \varepsilon\left(\int_{\Lambda_{3}}\left|\partial^{r-2} u\right|^{2} d x+\int_{\Lambda_{1}}\left|\partial^{r-1} u\right|^{2} d x\right)+\left.e(\varepsilon)\left|u \|_{L^{2}\left(\Lambda_{1}\right)}^{2}+\varepsilon^{-2\left(s_{1}-1\right)} \int_{\Lambda_{1}}\right| x\right|^{2\left(s_{1}-1\right)}\left|\partial^{r-1} u\right|^{2} d x \leqslant \\
\leqslant \varepsilon\|u\|_{W^{r-2}\left(A_{1}\right)}^{2}+\left(\varepsilon+\varepsilon^{-2\left(s_{1}-1\right)}\right) \int_{\Lambda_{1}}|x|^{2\left(s_{1}-1\right)}\left|\partial^{r-1} u\right|^{2} d x+e(\varepsilon)\|u\|_{L^{2}\left(A_{1}\right)}^{2}
\end{array}
$$

e da qui la (9.1) con $\lambda<1$. 
III) Sia $|\alpha|=r-2$.

Applichiamo la (1.7); si ha:

$$
\left|\partial^{r-2} u(0,0)\right|^{2} \leqslant \varepsilon\left(\int_{A_{1}}|x|^{2 s_{1}}\left|\partial^{r} u\right|^{2} d x+\int_{A_{1}}|x|^{2\left(s_{1}-1\right)}\left|\partial^{r-1} u\right|^{2} d x\right)+o(\varepsilon) \int_{A_{1}}|x|^{2 s_{1}}\left|\partial^{r-2} u\right|^{2} d x
$$

Osservando che il terzo integrale si maggiora con:

$$
\int_{\Lambda_{1}}\left|\partial^{x-2} u\right|^{2} d x
$$

ed applicando la diseguaglianza di Erling $\operatorname{con} \lambda=\varepsilon /(\varepsilon)$ si ricava l'asserto.

Lema 9.2. - Nelle ipotesi enunciate al n. 8 si ha per ogni $u \in W_{s_{1}, s_{2}}^{r}(A)$ :

$$
\|u\|_{U_{s_{1}, s_{2}}^{r}(A)} \leqslant c\left(\|\mathcal{A} u\|_{G_{r_{, s_{1}, s_{2}}(A)}}+\|u\|_{\left.\mathfrak{L}_{0, s_{2}-r-g^{2}(A)}^{2}\right)}\right)
$$

con $\varepsilon$ definito dall'ipotesi $\left.H_{3}^{\varepsilon}\right)$.

Siano $\zeta_{1}(x)$ e $\zeta_{2}(x)$ due funzioni di classe $D(\bar{\Lambda})$ tali che:

$$
\begin{array}{ll}
\zeta_{1}(x)=0 & \text { per }|x| \geqslant 2, \\
\zeta_{2}(x)=0 & \text { per }|x| \leqslant 1, \\
\zeta_{1}(x)+\zeta_{2}(x)=1 & \text { in } \bar{A} .
\end{array}
$$

Posto:

$$
u_{1}=\zeta_{1} \cdot u, \quad u_{2}=\zeta_{2} \cdot u
$$

riesce:

$$
u=u_{1}+u_{2}
$$

Per il Lemma 2.1, per la (1.14) di [3] e per la (6.4) di [1] si ha:

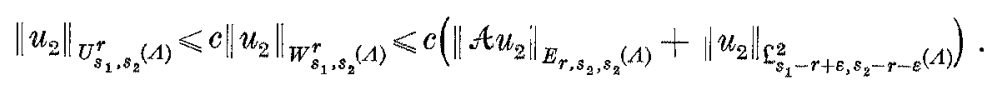

È quasi immediato che:

$$
\left\|u_{2}\right\|_{\mathfrak{S}_{s_{1}-r+\varepsilon, s_{1}-r-\varepsilon}^{2}(\lambda)} \leqslant e^{\prime}\|u\|_{\mathfrak{E}_{\mathbf{O}_{3}, s_{2}-r-\varepsilon}^{g}(\lambda)}
$$


Riesce inoltre per il Lemma 3.1:

$$
\begin{aligned}
& \left\|A u_{2}\right\|_{W_{s_{1}, s_{3}}^{r-2 m_{(A)}}} \leqslant c\left\|A u_{2}\right\|_{U_{s_{1}, z_{3}}^{r-2 m_{(A)}}} \leqslant \\
& \leqslant c\left(\left\|\zeta_{2} A u\right\|_{U_{\xi_{1}, \varepsilon_{2}}^{r-2 m}(\Lambda)}+\left\|\sum_{\mu \mid \leqslant 2 m} a_{\mu} \sum_{\alpha<\mu}(\mu) \partial_{\alpha}^{\mu-\alpha} \zeta_{2} \partial^{\alpha} u\right\|_{U_{s_{1}, s_{3}}^{\psi-2 m}(\Lambda)}\right) \leqslant \\
& \leqslant c^{\prime}\|A u\|_{s_{s_{1}, \varepsilon_{2}}^{r-2 m_{(A)}}}+e^{n} \lambda\|u\|_{W^{r}\left(\Lambda_{3}-A_{1}\right)}+e(\lambda)\|u\|_{L^{2}\left(A_{2}-A_{1}\right)} \leqslant \\
& \leqslant c^{\prime \prime}\left(\|A u\|_{U_{s_{1}, s_{2}}^{r-2 m}(\Lambda)}+\lambda\|u\|_{U_{s_{1}, s_{2}}^{\tau}(\Lambda)}+c(\lambda)\|u\|_{\mathcal{I}_{\delta_{2} s_{2}-r-l}^{2}}\right)
\end{aligned}
$$

dove si è applicata la diseguaglianza di Erling e si è tenuto conto che per $|\beta|>0$ supp $\left.\partial^{\beta} \zeta_{2} \subset A_{2}-A_{1}, \lambda \in\right] 0,1\left[, l \in R_{+}\right.$.

Analogamente si ha per il Lemma 4,2:

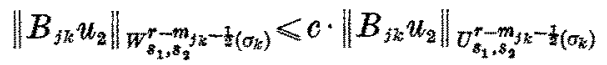

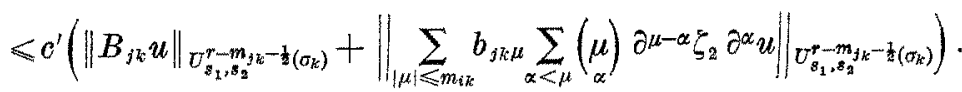

Per il Teorema 5.3 e dalle proprietà di $\zeta_{2}(x)$ si ha:

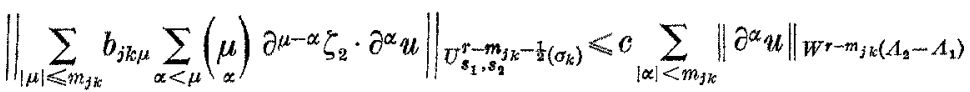

$$
\begin{aligned}
& \leqslant e^{\prime}\left(\lambda\|u\| W^{r}\left(A_{2}-A_{1}\right)+e(\lambda)\|u\|_{L^{2}\left(A_{2}-A_{1}\right)}\right) \\
& \leqslant c^{\prime \prime}\left(\lambda\|u\|_{U_{s_{1}, s_{2}}^{r}(A)}+c(\lambda)\|u\|_{\mathfrak{L}_{0, s_{2}-r-l}^{2}}\right) \\
& \operatorname{con} \lambda \in] 0,1\left[\text { ed } l \in R_{+}\right.
\end{aligned}
$$

Da quest'ultima e dalle $(9.3),(9.5),(9.6)$ segue:

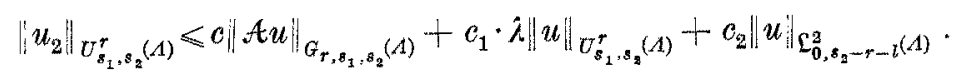

Per il Lemma 2.1, il Teorema 8.4 di [1] e per la (1.14) di [3] si ha per ogui $t_{2} \geqslant s_{1}$ :

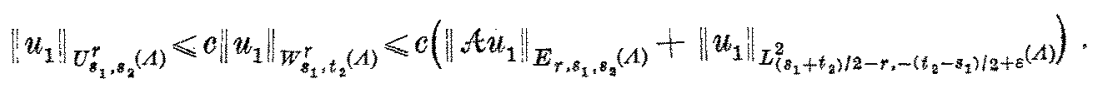

Per i Teoremi 8.2 e 8.6 di [1] e la (1.14) di [3], fissato $t_{2}$ in modo che riesca:

si ha:

$$
t_{2}-\varepsilon-r \geqslant s_{1}+\varepsilon-r
$$

$$
\begin{aligned}
& \left\|u_{1}\right\|_{L_{\left(s_{1}+\dot{t}_{2}\right) / 2-r,-\left(t_{2}-\xi_{1}\right) / 2+\varepsilon}^{2}(\Lambda)} \leqslant c\left\|u_{1}\right\|_{W_{s_{1}+\varepsilon-\tau, t_{2}-\varepsilon-\tau}^{0}(\Lambda)} \leqslant c_{1}\left\|u_{1}\right\|_{w_{s_{1}+\varepsilon, t_{2}-\varepsilon}^{\tau}(\Lambda)} \leqslant \\
& \leqslant c\left(\left\|\mathcal{A} u_{1}\right\|_{E_{r_{, 1}+s_{1}, t_{2}-\varepsilon}(A)}+\left\|u_{1}\right\|_{L_{\left(s_{1}+t_{2}\right) / 2-r,-\left(t_{2}-s_{1} / 2+2 \varepsilon\right.}^{2}(A)}\right) .
\end{aligned}
$$


Osserviamo ancora che per i Teoremi 8.4 e 8.2 di [1] riesce:

$$
\left\|\mathcal{A} u_{1}\right\|_{E_{r, s_{1}+\varepsilon_{,} t_{2}-\mathcal{E}}(A)} \leqslant c\left\|\mathcal{A} u_{1}\right\|_{E_{r, s_{1}+\varepsilon, t_{2}}(A)} \leqslant e^{\prime}\left\|\mathcal{A} u_{1}\right\|_{E_{r, s_{1}, s_{\varepsilon}}(A)}
$$

Iterando il procedimento si ha:

$$
\left\|u_{1}\right\|_{O_{3_{1}, s_{2}}^{r}(\Lambda)} \leqslant c\left(\left\|\mathcal{A} u_{1}\right\|_{E_{r, s_{1}, s_{2}}(A)}+\left\|u_{1}\right\|_{W_{s_{1}+k \varepsilon-r, t_{2}-k \varepsilon-r}(\Lambda)}\right),
$$

dove $k$ è tale che sia $s_{1}+k \varepsilon-r \geqslant 0$; si ha quindi:

$$
\left\|u_{1}\right\|_{W_{s_{1}+k \varepsilon-r, t_{2}-\bar{k} \varepsilon-r}^{0}(\Lambda)} \leqslant c\|u\|_{\mathfrak{L}_{0, s_{\mathrm{s}}-r-\varepsilon}(\Lambda)}
$$

Tenendo presente il Lemma 3.1 e maggiorando $\left\|\mathcal{A} u_{1}\right\|_{E r_{,}, s_{2}, s_{2}(\Lambda)}$ come nelle (9.5) e (9.6) si è fatto per $\left\|, \mathfrak{t}_{2} u\right\|_{E r, s_{1}, s_{2}(\Lambda)}$ si ha in definitiva:

$$
\left\|u_{1}\right\|_{U_{s_{1}, s_{2}}^{r}(A)} \leqslant c\left(\|\mathcal{A} u\|_{G_{r, s_{1}, s_{\varepsilon}}(A)}+\|u\|_{\mathfrak{L}_{\mathbb{Q}_{2}, s_{2}-r-\varepsilon}^{2}(A)}+e_{1} \cdot \lambda\|u\|_{U_{s_{1}, s_{2}}^{r}(A)}\right)
$$

con $\lambda \in] 0,1[$.

La (9.7) e la (9.10) danno la (9.2).

Poniamo, adesso, con $\grave{\mathrm{u}} \in U_{s_{3}, s_{2}}^{r}(\Lambda)$

$$
u^{\left(Q_{v}\right)}=u-\chi(x) \sum_{|\alpha| \leqslant p} \frac{\partial^{\alpha} u(0,0)}{\alpha \uparrow} x^{\alpha}
$$

dove $y=r-2-\left[s_{1}\right], \chi(x) \in \mathbb{D}(\bar{\Lambda}), \chi(x)=1$ per $|x| \leqslant 1, \chi(x)=0$ per $|x| \geqslant 2$.

Notiamo che in forza del Lemma 3.1, nelle ipotesi del n. 8 si ha:

$$
u^{\left(Q_{\nu}\right)} \in W_{s_{1}, s_{2}}^{r}(\Lambda)
$$

Dimostrazione del Teorema 8.1. - Osserviamo che - conservando le notazioni precedenti - si ha in forza del Lemma 9.1:

$$
\|u\|_{U_{s_{1}, s_{3}}^{r}(\Lambda)} \leqslant\left\|u^{\left(Q_{\nu}\right)}\right\|_{W_{s_{1}, s_{2}}^{r}(\Lambda)}+c \sum_{|\alpha| \leqslant \nu}\left|\partial^{\alpha} u(0,0)\right| \leqslant\left\|u^{\left(Q_{p}\right)}\right\|_{W_{s_{1}, s_{2}(\Lambda)}^{r}}+\lambda\|u\|_{U_{s_{1}, s_{\mathrm{g}}}^{r}(\Lambda)}+c(\lambda)\|u\|_{L^{g}\left(\Lambda_{1}\right)}
$$

cioè :

$$
\|u\|_{U_{s_{1}, s_{2}(A)}^{r}} \leqslant \frac{1}{1-\lambda}\left\|u^{\left(Q_{y}\right)}\right\|_{W_{s_{1}, s_{2}}^{r}(\Lambda)}+e^{\prime}(\lambda)\|u\|_{L^{2}\left(\Lambda_{1}\right)} .
$$

Da qui e dalla (9.2) segue:

$$
\|u\|_{U_{s_{1}, s_{3}}^{r}(\Lambda)} \leqslant c\left(\left\|\mathcal{A} u^{\left(Q_{v}\right)}\right\|_{G_{r, s_{1}, s_{2}}(\Lambda)}+\left\|u^{\left(Q_{v}\right)}\right\|_{\mathfrak{L}_{0, s_{2}-r-\mathcal{E}}^{2}(\Lambda)}+\|u\|_{L^{2}\left(\Lambda_{1}\right)}\right) .
$$


Osserviamo che:

$$
\begin{aligned}
& \left\|A u^{\left(Q_{\nu}\right)}\right\|_{U_{s_{1}, s_{2}}^{r-2 m}(\Lambda)} \leqslant\|A u\|_{U_{s_{1}, s_{2}}^{r-2 m_{2}(\Lambda)}}+\left\|A\left(\chi Q_{v}\right)\right\|_{U_{s_{1}, s_{2}}^{r-2 m_{2}}(\Lambda)} \leqslant c\left(\|A u\|_{U_{s_{1}, s_{2}}^{r-2 m_{2}(\Lambda)}}+\sum_{|\alpha| \leqslant v} \mid \partial^{\alpha} u(0,0) !\right), \\
& \left\|B_{j k} u^{\left(Q_{y}\right)}\right\|_{U_{s_{1}, s_{\mathrm{g}}}^{r-m_{j k}-\frac{1}{2}\left(\sigma_{k}\right)}} \leqslant\left\|B_{j k} u\right\|_{U_{s_{1}, s_{2}}^{r-m_{j k}-\frac{1}{2}\left(\sigma_{k}\right)}}+\left\|B_{j k}\left(\chi Q_{\nu}\right)\right\|_{U_{s_{1}, s_{\mathrm{g}}}^{r-m_{j k}-\frac{1}{2}\left(\sigma_{k}\right)}},
\end{aligned}
$$

ed in virtù del Teorema 5.3 che:

$$
\left.\left\|B_{j k}\left(\chi Q_{\nu}\right)\right\|_{U_{s_{1}, s_{2}}^{r-m_{j k}-\frac{1}{2}\left(\sigma_{k}\right)}} \leqslant c \sum_{\left\{\mu \mid \leqslant m_{j k}\right.}\left\|\dot{b}_{j k \mu} \partial^{\mu}\left(\chi Q_{\nu}\right)\right\|_{U_{s_{1}, s_{2}}^{r-m_{j k}(\Lambda)}} \leqslant e^{\prime} \sum_{\left\{\alpha_{i}^{*} \leqslant \nu\right.} \mid \partial^{\alpha} u(0,0)\right\}
$$

Inoltre si ha:

$$
\begin{aligned}
& \left\|u^{\left(Q_{\nu}\right)}\right\|_{\mathfrak{L}_{\mathbf{0}_{,}, s_{2}-r-\varepsilon}^{2}(A)} \leqslant\|u\|_{\mathfrak{L}_{\mathbf{0}_{0}, s_{2}-r-\varepsilon}(\Lambda)}+c \sum_{|\alpha| \leqslant \nu}\left|\partial^{\alpha} u(0,0)\right|, \\
& \|u\|_{L^{2}\left(\Lambda_{1}\right)} \leqslant\|u\|_{\mathbb{L}_{0, s_{z}-r-\varepsilon}^{2}(\Lambda)}
\end{aligned}
$$

Da quest'ultima diseguaglianza, dalla (9.11) e dalla (9.1) segue la (8.1).

\section{0. - Il problema aggiunto.}

Indicato con $G_{r, s_{1}, s_{2}}^{\prime}(\Lambda)$ e $\left(U_{s_{1}, s_{2}}^{r}(\Lambda)\right)^{\prime}$ i duali forti di $G_{r, s_{1}, s_{2}}(\Lambda)$ e $U_{s_{1}, s_{2}}^{r}(\Lambda)$, consideriamo l'operatore $\mathcal{A}^{*}$ aggiunto funzionale di $\mathcal{A}$, lineare e continuo da $G_{r, s_{1}, s_{2}}^{\prime}(A)$ in $\left(U_{s_{1}, s_{2}}^{r}(\Lambda)\right)^{\prime}$, definito dalla relazione:

$$
\langle v, \mathfrak{A} u\rangle=\left\langle\mathfrak{A}^{*} v, u\right\rangle .
$$

$\forall u \in U_{s_{1}, s_{2}}^{r}(\Lambda)$ e $v \equiv\left(\vartheta, \vartheta_{11}, \ldots, \vartheta_{m 2}\right) \in G_{r, s_{1}, s_{2}}^{\prime}(A)$

Supponiamo d'ora in poi che oltre alle ipotesi del n. 8 sia verificata la seguente:

$\left.H_{4}^{\varepsilon}\right)$ esiste un $\varepsilon \geqslant 0$ tale che:

$$
\begin{aligned}
& |x|^{2 m-|\mu|+|\alpha|+\varepsilon} \partial^{\alpha} a_{\mu} \in L^{\infty}\left(A-A_{1}\right)\left\{\begin{array}{l}
|\mu| \leqslant 2 m-1, \\
|\alpha| \leqslant r+1-2 m,
\end{array}\right. \\
& |x|^{\left|m_{j k}-\right| \mu|+| \alpha \mid+8} \partial^{\alpha} b_{j k \mu}^{\circ} \in L^{\infty}\left(A-A_{1}\right)\left\{\begin{array}{l}
|\mu| \leqslant m_{j k}-1, \\
|\alpha| \leqslant r+1-m_{j k} .
\end{array}\right.
\end{aligned}
$$

Vale il seguente: 
Teorema 10.1. - Nelle ipotesi poste con $s_{1}-r+1 \notin \Sigma_{1}, s_{2}-r+1 \notin \Sigma_{2}$ con $r \neq$ $\neq 2 m+2$ e $r \neq m_{j k}+2$ se $s_{1}>1$, con $s_{1} \neq \varepsilon, s_{1} \leqslant \frac{1}{2}+\varepsilon$ e con $\varepsilon$ definito dall'ipotesi $H_{4}^{e}$ ), vale la seguente maggiorazione:

$$
\begin{gathered}
\|v\|_{G_{r, s_{1}, s_{2}}^{\prime}(A)} \leqslant k\left(\left\|\mathcal{A}^{*} v\right\|_{\left(U_{s_{1}, s_{2}}^{r}(A)\right)^{\prime}}+\|v\|_{G_{r+1, s_{1}+1-\varepsilon, s_{2}+1+\varepsilon}^{\prime}(A)}\right) \\
\left.\forall v \equiv\left(\vartheta, \vartheta_{11}, \ldots, \vartheta_{m, 2}\right) \in G_{r, s_{1}, s_{2}}^{\prime} \Lambda\right) .
\end{gathered}
$$

Sia $\vartheta \in\left(U_{s_{1}, s_{2}}^{r-2 m}(\Lambda)\right)^{r}$ ed $r \geqslant 2 m+2$.

Si ha $\forall u \in U_{s_{1}, s_{2}}^{r-2 m}(\Lambda)$ con $y=r-2 m-2-\left[s_{1}\right]$ :

$$
\left\langle\vartheta, u-\chi(x) \sum_{|y| \leqslant v} \frac{\partial^{\gamma} u(0,0)}{\gamma !} x^{\gamma}\right\rangle=\langle\vartheta, u\rangle-\sum_{|\vartheta| \leqslant \vartheta} \frac{\partial^{\gamma} u(0,0)}{\gamma !}\left\langle\vartheta, \chi^{\left.x^{y}\right\rangle}\right\rangle
$$

dove $\chi(x)$ è la solita funzione di classe $\mathfrak{D}(\bar{\Lambda})$.

Quindi:

$$
\begin{aligned}
& \|\vartheta\|_{\left(U_{s_{1}, s_{1}}^{r-2 m}(\Lambda)\right)^{\prime}}=\sup _{u \in U_{s_{1}, s_{3}}^{r-2 m}(\Lambda)} \frac{|\langle\vartheta, u\rangle|}{\|u\|_{U_{s_{1}, s_{3}}^{r-2 m}(\Lambda)}}
\end{aligned}
$$

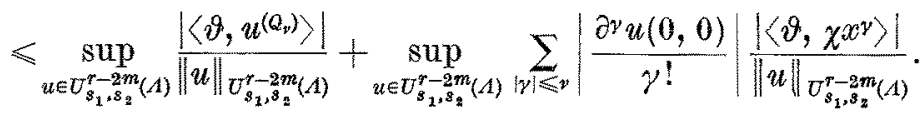

D'altro canto:

$$
W_{s_{1}, s_{2}}^{r-2 m}(\Lambda) \subset U_{s_{1}, s_{1}}^{r-2 m}(\Lambda) \Rightarrow\left(U_{s_{1}, s_{3}}^{r-2 m}(\Lambda)\right)^{\prime} \subset\left(W_{s_{1}, s_{2}}^{r-2 m}(\Lambda)\right)^{\prime}
$$

ed inoltre per il Lemma 3.1 riesce $u^{\left(Q_{x}\right)} \in W_{s_{1}, s_{2}}^{r-2 m}(\Lambda)$.

Quindi

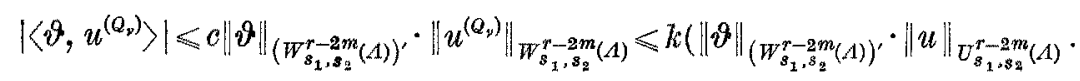

D'altro eanto per il Lemma 6.3 si ha:

$$
\left|\left\langle\vartheta, \chi x x^{\gamma}\right\rangle\right| \leqslant c\|\vartheta\|_{\left(U_{s_{1}+1-\varepsilon, s_{2}+1+\varepsilon}^{++1-2 m}(\Lambda)\right)^{\prime}} \cdot\left\|\chi x^{\gamma}\right\|_{U_{s_{1}+1-s_{2}+s_{2}+1+\varepsilon}^{r+1-2 m}} \cdot
$$

Da qui, dalle (10.2) e (10.3) e dall'osservazione che:

$$
\sum_{|\gamma| \leqslant p}\left|\frac{\partial^{y} u(0,0)}{\gamma !}\right| \leqslant c\|u\|_{U_{s_{1}, s_{2}}^{r-2 m_{(-1)}},},
$$

segue che:

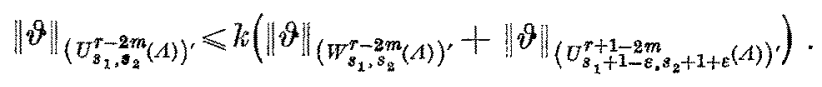


Sia adesso $\vartheta_{j k} \in\left(U_{s_{1}, s_{z}}^{r-m_{k}-\frac{1}{2}}\left(\sigma_{k}\right)\right)^{\prime} . \forall g \in U_{s_{1}, s_{s}}^{r-m_{j k}-\frac{1}{3}}\left(\sigma_{k k}\right)$ riesce:

$$
\left\langle\vartheta_{j k}, g-\chi(\varrho) \sum_{\gamma \leqslant \nu} \frac{\partial^{\gamma} g(0)}{\gamma !} \varrho^{\gamma}\right\rangle=\left\langle\vartheta_{i k}, g\right\rangle-\sum_{\gamma \leqslant \nu} \frac{\partial^{\gamma} g(0)}{\gamma !}\left\langle\vartheta_{j k}, \chi(\varrho) \varrho^{\gamma}\right\rangle,
$$

con

$$
y=r-m_{j k}-2-\left[s_{1}\right] \quad \text { e } \quad \chi \in \mathscr{D}\left(\left[0 , + \infty [ ) \quad \text { con } \quad \chi \left\{\begin{array}{ll}
=1 & \text { per } \varrho \leqslant 1 \\
=0 & \text { per } \varrho \geqslant 2 .
\end{array}\right.\right.\right.
$$

Si ha:

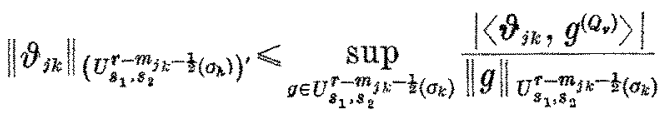

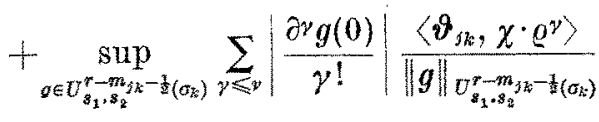

ove si è posto:

$$
g^{\left(Q_{\nu}\right)}=g-\chi \sum_{\gamma \leqslant \nu} \frac{\partial^{\gamma} g(0)}{\gamma !} Q^{\gamma}
$$

Si ha procedendo come nel caso precedente:

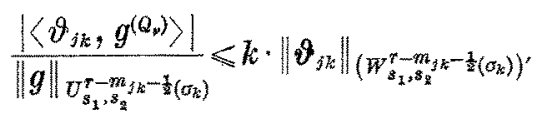

ed in virtù del Lemmi 6.5 e 6.6 :

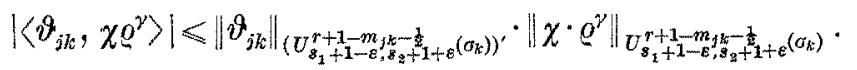

In definitiva da $(10.5),(10.6),(10.7)$ segue:

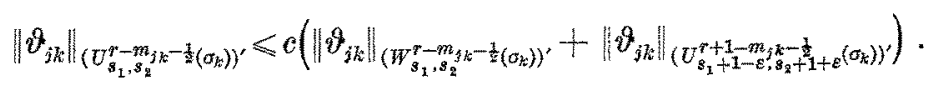

Dalla $(10.4)$ e $(10.8)$ segue ancora:

$$
\|v\|_{G_{r, s_{1}, s_{g}}^{\prime}(L)} \leqslant k\left(\|v\|_{E_{r, s_{1}, s_{\mathrm{g}}}^{\prime}(\Lambda)}+\|v\|_{a_{r+1, s_{1}+1-\varepsilon, s_{2}+1+\varepsilon}^{\prime}(\Lambda)}\right)
$$

e tenendo conto della (1.16) di [3] si ha:

$$
\|v\|_{G_{r, s_{1}, s_{2}}^{\prime}(\Lambda)} \leqslant k^{\prime}\left(\left\|\mathcal{A}^{*} v\right\|_{\left(W_{s_{1}, s_{2}}^{\tau}(\Lambda)\right)^{\prime}}+\|v\|_{E_{r+1, s_{1}+1-\mathcal{\xi}, s_{2}+1+\varepsilon}^{\prime}(\Lambda)}+\|v\|_{G_{r+1, s_{1}+1-\varepsilon, s_{2}+1+\varepsilon^{\prime}}^{\prime}(\Lambda)}\right) .
$$


D'altro canto poichè:

$$
\left(W_{s_{1}, s_{2}}^{r}(\Lambda)\right)^{\prime} \supset\left(U_{s_{1}, s_{2}}^{r}(\Lambda)\right)^{\prime}, \quad G_{r+1, s_{1}+1-\varepsilon, s_{2}+1+\varepsilon}^{\prime}(A) \subset E_{r+1, s_{1}+1-\varepsilon, s_{2}+1+\varepsilon}^{\prime}(B)
$$

si ha la (10.1).

Dal Corollario 8.1, dal Teorema 10.1, dai Lemmi 7.1 e 7.2 e dal Lemma 5.1, pag. 171 di [5] segue il:

Teorema 10.2. - Nelle ipotesi del Teorema 10.1 con $\varepsilon>0$ e con $\left.s_{1} \in\right] 0,1[$ l'operatore $\mathfrak{A} \dot{e}$ ad indice.

\section{BIBLIOGRAFIA}

[1] A. Avantaggtatr - M. Trorsr, Spazi di Sobolev con peso e problemi ellittici in un angolo, $I$, Ann. di Mat. pura e appl., 95 (1973), pp. 361-408.

[2] A. Avantaggiati - M. Troisi, Spazi di Sobolev con peso e problemi ellittici in un angolo, II, Ann. di Mat. pura e appl., 97 (1973), pp. 207-252.

[3] A. Avantaggiati - M. Troisi, Spazi di Sobolev con peso e problemi ellittici in un angolo III, Ann. di Mat. pura e appl., 99 (1974), pp. 1-51.

[4] P. GRISvard, Altemative de Fredholm relative au problème de Dirichlet dans un polygone ou un polyèdre, Boll. U.M.I., 5 (1972), pp. 132-164.

[5] J. L. Lrons - E. Magenes, Problèmes aux limites non homogènes, vol. 1, Dunod, Paris (1968).

[6] J. NæČAs, Les méthodes directes en théorie des équations elliptiques, Masson et Cie, Editeurs, Paris; Acad. Editeurs, Prague (1967). 